Patrick Bernard · Boris Buffoni

\title{
Optimal mass transportation and Mather theory
}

Received July 20, 2005

Abstract. We study the Monge transportation problem when the cost is the action associated to a Lagrangian function on a compact manifold. We show that the transportation can be interpolated by a Lipschitz lamination. We describe several direct variational problems the minimizers of which are these Lipschitz laminations. We prove the existence of an optimal transport map when the transported measure is absolutely continuous. We explain the relations with Mather's minimal measures.

Several observations have recently renewed the interest for the classical topic of optimal mass transportation, whose origin is attributed to Monge a few years before the French revolution. The framework is as follows. A space $M$ is given, which in the present paper will be a compact manifold, as well as a continuous cost function $c(x, y): M \times M \rightarrow \mathbb{R}$. Given two probability measures $\mu_{0}$ and $\mu_{1}$ on $M$, the mappings $\Psi: M \rightarrow M$ which transport $\mu_{0}$ into $\mu_{1}$ and minimize the total cost $\int_{M} c(x, \Psi(x)) d \mu_{0}$ are studied. It turns out, and it was the core of the investigations of Monge, that these mappings have very remarkable geometric properties, at least at a formal level.

Only much more recently was the question of the existence of optimal objects rigorously solved by Kantorovich in a famous paper of 1942. Here we speak of optimal objects, and not of optimal mappings, because the question of existence of an optimal mapping is ill-posed, so that the notion of optimal objects has to be relaxed, in a way that nowadays seems very natural, and that was discovered by Kantorovich.

Our purpose here is to continue the work initiated by Monge, recently awakened by Brenier and enriched by other authors, on the study of geometric properties of optimal objects. The cost functions we consider are natural generalizations of the $\operatorname{cost} c(x, y)=$ $d(x, y)^{2}$ considered by Brenier and many other authors. The book [35] gives some ideas on the applications expected from this kind of questions. More precisely, we consider a Lagrangian function $L(x, v, t): T M \times \mathbb{R} \rightarrow \mathbb{R}$ which is convex in $v$ and satisfies standard

P. Bernard: Institut Fourier, Grenoble, CEREMADE, Université de Paris Dauphine, Pl. du Maréchal de Lattre de Tassigny, 75775 Paris Cedex 16, France;

e-mail: patrick.bernard@ceremade.dauphine.fr

B. Buffoni: School of Mathematics, École Polytechnique Fédérale-Lausanne, SB/IACS/ANA

Station 8, 1015 Lausanne, Switzerland; e-mail: boris.buffoni@epfl.ch 
hypotheses recalled later, and define our cost by

$$
c(x, y)=\min _{\gamma} \int_{0}^{1} L(\gamma(t), \dot{\gamma}(t), t) d t
$$

where the minimum is taken over the set of curves $\gamma:[0,1] \rightarrow M$ satisfying $\gamma(0)=x$ and $\gamma(1)=y$. Note that this class of costs does not contain the very natural cost $c(x, y)=$ $d(x, y)$. Such costs are studied in another paper [8].

Our main result is that the optimal transports can be interpolated by measured Lipschitz laminations, or geometric currents in the sense of Ruelle and Sullivan. Interpolations of transport have already been considered by Benamou, Brenier and McCann for less general cost functions, and with different purposes. Our methods are inspired by the theory of Mather, Mañé and Fathi on Lagrangian dynamics, and we will detail rigorously the relations between these theories. Roughly, they are exactly similar except that mass transportation is a Dirichlet boundary value problem, while Mather theory is a periodic boundary value problem. We will also prove, extending work of Brenier, Gangbo, McCann, Carlier, and others, that the optimal transportation can be performed by a Borel map with the additional assumption that the transported measure is absolutely continuous.

Various connections between Mather-Fathi theory, optimal mass transportation and Hamilton-Jacobi equations have recently been discussed, mainly at a formal level; see for example [35], or [18], where they are all presented as infinite-dimensional linear programming problems. This has motivated a lot of activity around the interface between Aubry-Mather theory and optimal transportation, some of which overlaps partly the present work. For example, at the moment of submitting the paper, we were informed about recent preprints of De Pascale, Gelli and Granieri [14] and of Granieri [25]. We had also been aware of a manuscript by Wolansky [36], which, independently, and by somewhat different methods, obtains results similar to ours. Note however that Lipschitz regularity, which we consider one of our most important results, was not obtained in this preliminary version of [36]. The papers [32] of Pratelli and [28] of Loeper are also worth mentioning.

\section{Introduction}

We present the context and the main results of the paper.

\subsection{Lagrangian, Hamiltonian and cost}

In all the present paper, the space $M$ will be a compact and connected Riemannian manifold without boundary. Some standing notations are gathered in the appendix. Let us fix a positive real number $T$, and a Lagrangian function

$$
L \in C^{2}(T M \times[0, T], \mathbb{R}) .
$$


A curve $\gamma \in C^{2}([0, T], M)$ is called an extremal if it is a critical point of the action

$$
\int_{0}^{T} L(\gamma(t), \dot{\gamma}(t), t) d t
$$

with fixed endpoints. It is called a minimizing extremal if it minimizes the action. We assume:

- Convexity: for each $(x, t) \in M \times[0, T]$, the function $v \mapsto L(x, v, t)$ is convex with positive definite Hessian at each point.

- Superlinearity: for each $(x, t) \in M \times[0, T], L(x, v, t) /\|v\| \rightarrow \infty$ as $\|v\| \rightarrow \infty$. Arguing as in [19, Lemma 3.2.2], this implies that for all $\alpha>0$ there exists $C>0$ such that $L(x, v, t) \geq \alpha\|v\|-C$ for all $(x, v, t) \in T M \times[0, T]$.

- Completeness: for each $(x, v, t) \in T M \times[0, T]$, there exists a unique extremal $\gamma \in$ $C^{2}([0, T], M)$ such that $(\gamma(t), \dot{\gamma}(t))=(x, v)$.

We associate to the Lagrangian $L$ a Hamiltonian function $H \in C^{2}\left(T^{*} M \times[0, T], \mathbb{R}\right)$ given by

$$
H(x, p, t)=\max _{v}(p(v)-L(x, v, t)) .
$$

We endow the cotangent bundle $T^{*} M$ with its canonical symplectic structure, and associate to the Hamiltonian $H$ the time-dependent vector field $Y$ on $T^{*} M$, given by

$$
Y=\left(\partial_{p} H,-\partial_{x} H\right)
$$

in any canonical local trivialization of $T^{*} M$. The hypotheses on $L$ can be expressed in terms of the function $H$ :

- Convexity: for each $(x, t) \in M \times[0, T]$, the function $p \mapsto H(x, p, t)$ is convex with positive definite Hessian at each point.

- Superlinearity: for each $(x, t) \in M \times[0, T]$, we have $H(x, p, t) /\|p\| \rightarrow \infty$ as $\|p\| \rightarrow \infty$.

- Completeness: each solution of the equation $(\dot{x}(t), \dot{p}(t))=Y(x(t), p(t), t)$ can be extended to the interval $[0, T]$. We can then define, for all $s, t \in[0, T]$, the flow $\varphi_{s}^{t}$ of $Y$ from time $s$ to time $t$.

In addition, the mapping $\partial_{v} L: T M \times[0, T] \rightarrow T^{*} M \times[0, T]$ is a $C^{1}$ diffeomorphism, whose inverse is the mapping $\partial_{p} H$. These diffeomorphisms conjugate $Y$ to a time-dependent vector field $E$ on $T M$. We denote the flow of $E$ by $\psi_{s}^{t}: T M \rightarrow T M$ $(s, t \in[0, T])$; it satisfies $\psi_{s}^{s}=\operatorname{Id}$ and $\partial_{t} \psi_{s}^{t}=E_{t} \circ \psi_{s}^{t}$, where as usual $E_{t}$ denotes the vector field $E(\cdot, t)$ on $T M$. The diffeomorphisms $\partial_{v} L$ and $\partial_{p} H$ conjugate the flows $\psi_{s}^{t}$ and $\varphi_{s}^{t}$. Moreover the extremals are the projections of the integral curves of $E$ and

$$
\left(\pi \circ \psi_{s}^{t}, \partial_{t}\left(\pi \circ \psi_{s}^{t}\right)\right)=\psi_{s}^{t},
$$

where $\pi: T M \rightarrow M$ is the canonical projection. In (1), $\partial_{t}\left(\pi \circ \psi_{s}^{t}\right)$ is seen as a vector in the tangent space of $M$ at $\pi \circ \psi_{s}^{t}$. If $\partial_{t}\left(\pi \circ \psi_{s}^{t}\right)$ is seen as a point in $T M$, (1) becomes simply $\partial_{t}\left(\pi \circ \psi_{s}^{t}\right)=\psi_{s}^{t}$. 
For each $0 \leq s<t \leq T$, we define the cost function

$$
c_{s}^{t}(x, y)=\min _{\gamma} \int_{s}^{t} L(\gamma(\sigma), \dot{\gamma}(\sigma), \sigma) d \sigma
$$

where the minimum is taken over the set of curves $\gamma \in C^{2}([s, t], M)$ satisfying $\gamma(s)=x$ and $\gamma(t)=y$. That this minimum exists is a standard result under our hypotheses (see [30] or [19]).

Proposition 1. Fix a subinterval $[s, t] \subset[0, T]$. The set $\mathcal{E} \subset C^{2}([s, t], M)$ of minimizing extremals is compact for the $C^{2}$ topology.

Let us mention that, for each $\left(x_{0}, s\right) \in M \times[0, T]$, the function $(x, t) \mapsto c_{s}^{t}\left(x_{0}, x\right)$ is a viscosity solution of the Hamilton-Jacobi equation

$$
\partial_{t} u+H\left(x, \partial_{x} u, t\right)=0
$$

on $M \times] s, T$. This remark may help the reader understand the key role which will be played by this equation in what follows.

\subsection{Monge-Kantorovich theory}

We recall the basics of Monge-Kantorovich duality. The proofs are available in many texts on the subject, for example [1, 33, 35]. We assume that $M$ is a compact manifold and that $c$ is a continuous cost function on $M \times M$, which will later be one of the $\operatorname{costs} c_{s}^{t}$ defined above. Given two Borel probability measures $\mu_{0}$ and $\mu_{1}$ on $M$, a transport plan between $\mu_{0}$ and $\mu_{1}$ is a measure $\eta$ on $M \times M$ which satisfies

$$
\left(\pi_{0}\right)_{\sharp}(\eta)=\mu_{0} \quad \text { and } \quad\left(\pi_{1}\right)_{\sharp}(\eta)=\mu_{1},
$$

where $\pi_{0}: M \times M \rightarrow M$ is the projection on the first factor, and $\pi_{1}$ is the projection on the second factor. We denote by $\mathcal{K}\left(\mu_{0}, \mu_{1}\right)$, after Kantorovich, the set of transport plans. Kantorovich proved the existence of the minimum

$$
C\left(\mu_{0}, \mu_{1}\right)=\min _{\eta \in \mathcal{K}\left(\mu_{0}, \mu_{1}\right)} \int_{M \times M} c d \eta
$$

for each pair $\left(\mu_{0}, \mu_{1}\right)$ of probability measures on $M$. Here we will denote by

$$
C_{s}^{t}\left(\mu_{0}, \mu_{1}\right):=\min _{\eta \in \mathcal{K}\left(\mu_{0}, \mu_{1}\right)} \int_{M \times M} c_{s}^{t}(x, y) d \eta(x, y)
$$

the optimal value associated to our family of costs $c_{s}^{t}$ The plans which realize this minimum are called optimal transfer plans. A pair $\left(\phi_{0}, \phi_{1}\right)$ of continuous functions is called an admissible Kantorovich pair if it satisfies the relations

$$
\phi_{1}(x)=\min _{y \in M}\left(\phi_{0}(y)+c(y, x)\right) \quad \text { and } \quad \phi_{0}(x)=\max _{y \in M}\left(\phi_{1}(y)-c(x, y)\right)
$$


for all $x \in M$. Note that the admissible pairs are composed of Lipschitz functions if the cost $c$ is Lipschitz, which is the case of the costs $c_{s}^{t}$ when $s<t$. Another discovery of Kantorovich is that

$$
C\left(\mu_{0}, \mu_{1}\right)=\max _{\phi_{0}, \phi_{1}}\left(\int_{M} \phi_{1} d \mu_{1}-\int_{M} \phi_{0} d \mu_{0}\right)
$$

where the maximum is taken over the set of admissible Kantorovich pairs $\left(\phi_{0}, \phi_{1}\right)$. This maximization problem is called the dual Kantorovich problem, and the admissible pairs which reach this maximum are called optimal Kantorovich pairs. The direct problem (2) and dual problem (3) are related as follows.

Proposition 2. If $\eta$ is an optimal transfer plan, and if $\left(\phi_{0}, \phi_{1}\right)$ is an optimal Kantorovich pair, then the support of $\eta$ is contained in the set

$$
\left\{(x, y) \in M^{2}: \phi_{1}(y)-\phi_{0}(x)=c(x, y)\right\} .
$$

Let us remark that the knowledge of the set of admissible Kantorovich pairs is equivalent to the knowledge of the cost function $c$.

Lemma 3. We have

$$
c(x, y)=\max _{\left(\phi_{0}, \phi_{1}\right)}\left(\phi_{1}(y)-\phi_{0}(x)\right)
$$

where the maximum is taken over the set of admissible Kantorovich pairs.

Proof. This maximum clearly does not exceed $c(x, y)$. For the other inequality, fix $x_{0}$ and $y_{0}$ in $M$, and consider the functions $\phi_{1}(y)=c\left(x_{0}, y\right)$ and $\phi_{0}(x)=\max _{y \in M}\left(\phi_{1}(y)-\right.$ $c(x, y))$. We have $\phi_{1}\left(y_{0}\right)-\phi_{0}\left(x_{0}\right)=c\left(x_{0}, y_{0}\right)-0=c\left(x_{0}, y_{0}\right)$. So it is enough to prove that $\left(\phi_{0}, \phi_{1}\right)$ is an admissible Kantorovich pair, and more precisely that $\phi_{1}(y)=$ $\min _{x \in M}\left(\phi_{0}(x)+c(x, y)\right)$. We have

$$
\phi_{0}(x)+c(x, y) \geq c\left(x_{0}, y\right)-c(x, y)+c(x, y) \geq c\left(x_{0}, y\right)=\phi_{1}(y),
$$

which gives the inequality $\phi_{1}(y) \leq \min _{x \in M}\left(\phi_{0}(x)+c(x, y)\right)$. On the other hand, we have

$$
\min _{x \in M}\left(\phi_{0}(x)+c(x, y)\right) \leq \phi_{0}\left(x_{0}\right)+c\left(x_{0}, y\right)=c\left(x_{0}, y\right)=\phi_{1}(y) .
$$

\subsection{Interpolations}

In this section, the Lagrangian $L$ and time $T>0$ are fixed. It is not hard to see that if $\mu_{1}, \mu_{2}$ and $\mu_{3}$ are three probability measures on $M$, and if $t_{1} \leq t_{2} \leq t_{3} \in[0, T]$, then

$$
C_{t_{1}}^{t_{3}}\left(\mu_{1}, \mu_{3}\right) \leq C_{t_{1}}^{t_{2}}\left(\mu_{1}, \mu_{2}\right)+C_{t_{2}}^{t_{3}}\left(\mu_{2}, \mu_{3}\right) .
$$

The family $\mu_{t}, t \in[0, T]$, of probability measures on $M$ is called an interpolation between $\mu_{0}$ and $\mu_{T}$ if

$$
C_{t_{1}}^{t_{3}}\left(\mu_{t_{1}}, \mu_{t_{3}}\right)=C_{t_{1}}^{t_{2}}\left(\mu_{t_{1}}, \mu_{t_{2}}\right)+C_{t_{2}}^{t_{3}}\left(\mu_{t_{2}}, \mu_{t_{3}}\right)
$$

for all $0 \leq t_{1} \leq t_{2} \leq t_{3} \leq T$. Our main result is the following: 
Theorem A. For each pair $\mu_{0}, \mu_{T}$ of probability measures, there exist interpolations between $\mu_{0}$ and $\mu_{T}$. Moreover, each interpolation $\mu_{t}, t \in[0, T]$, is given by a Lipschitz. measured lamination in the following sense:

Eulerian description: There exists a bounded locally Lipschitz vector field $X(x, t)$ : $M \times] 0, T\left[\rightarrow T M\right.$ such that, if $\left.\Psi_{s}^{t},(s, t) \in\right] 0, T\left[{ }^{2}\right.$, is the flow of $X$ from time $s$ to time $t$, then $\left(\Psi_{s}^{t}\right)_{\sharp} \mu_{s}=\mu_{t}$ for each $\left.(s, t) \in\right] 0, T\left[^{2}\right.$.

Lagrangian description: There exists a family $\mathcal{F} \subset C^{2}([0, T], M)$ of minimizing extremals $\gamma$ of $L$ such that $\dot{\gamma}(t)=X(\gamma(t), t)$ for all $t \in] 0, T[$ and $\gamma \in \mathcal{F}$. The set

$$
\tilde{\mathcal{T}}=\{(\gamma(t), \dot{\gamma}(t), t): t \in] 0, T[, \gamma \in \mathcal{F}\} \subset T M \times] 0, T[
$$

is invariant under the Euler-Lagrange flow $\psi$. The measure $\mu_{t}$ is supported on $\mathcal{T}_{t}=$ $\{\gamma(t): \gamma \in \mathcal{F}\}$. In addition, there exists a continuous family $m_{t}, t \in[0, T]$, of probability measures on $T M$ such that $m_{t}$ is concentrated on $\tilde{\mathcal{T}}_{t}=\{(\gamma(t), \dot{\gamma}(t)): \gamma \in \mathcal{F}\}$ for each $t \in] 0, T\left[\pi_{\sharp} m_{t}=\mu_{t}\right.$ for each $t \in[0, T]$, and

$$
m_{t}=\left(\psi_{s}^{t}\right)_{\sharp} m_{S} \quad \text { for all }(s, t) \in[0, T]^{2} .
$$

Hamilton-Jacobi equation: There exists a Lipschitz $C^{1}$ function $\left.v: M \times\right] 0, T[\rightarrow \mathbb{R}$ which satisfies

$$
\partial_{t} v+H\left(x, \partial_{x} v, t\right) \leq 0,
$$

with equality if and only if $(x, t) \in \mathcal{T}=\{(\gamma(t), t): \gamma \in \mathcal{F}, t \in] 0, T[\}$, and such that $X(x, t)=\partial_{p} H\left(x, \partial_{x} v(x, t), t\right)$ for each $(x, t) \in \mathcal{T}$.

Uniqueness: There may exist several different interpolations. However, one can choose the vector field $X$, the family $\mathcal{F}$ and the subsolution $v$ in such a way that the statements above hold for all interpolations $\mu_{t}$ with these fixed $X, \mathcal{F}$ and $v$. For each $\left.s<t \in\right] 0, T[$, the measure $\left(\operatorname{Id} \times \Psi_{s}^{t}\right)_{\sharp} \mu_{s}$ is the only optimal transport plan in $\mathcal{K}\left(\mu_{s}, \mu_{t}\right)$ for the cost $c_{s}^{t}$. This implies that

$$
\int_{M} c_{s}^{t}\left(x, \Psi_{s}^{t}(x)\right) d \mu_{s}(x)=C_{s}^{t}\left(\mu_{s}, \mu_{t}\right)
$$

Let us comment on the preceding statement. The set $\tilde{\mathcal{T}} \subset T M \times] 0, T$ [ is the image under the Lipschitz map $(x, t) \mapsto(X(x, t), t)$ of the set $\mathcal{T} \subset T M \times] 0, T[$. We shall not take $X(x, t)=\partial_{p} H\left(x, \partial_{x} v(x, t), t\right)$ outside of $\mathcal{T}$ because we do not prove that this vector field is Lipschitz outside of $\mathcal{T}$. The data of the vector field $X$ outside of $\mathcal{T}$ is immaterial: any Lipschitz extension of $X_{\mid \mathcal{T}}$ will do. Note also that the relation

$$
\Psi_{s}^{t}=\pi \circ \psi_{s}^{t} \circ X_{s}
$$

holds on $\mathcal{T}_{s}$, where $X_{s}(\cdot)=X(\cdot, s)$.

The vector field $X$ in the statement depends on the transported measures $\mu_{0}$ and $\mu_{T}$. The Lipschitz constant of $X$, however, can be fixed independently of these measures, as we now state (see Proposition 13, Proposition 19, Theorem 3 and (11)): 
Addendum. There exists a decreasing function $K(\epsilon):] 0, T / 2[\rightarrow] 0, \infty[$, which depends only on the time $T$ and on the Lagrangian $L$, such that, for each pair $\mu_{0}, \mu_{T}$ of probability measures, one can choose the vector field $X$ in Theorem $A$ in such a way that $X$ is $K(\epsilon)$-Lipschitz on $M \times[\epsilon, T-\epsilon]$ for each $\epsilon \in] 0, T / 2[$.

Proving Theorem A is the main goal of the present paper. In Section 2 we will present some direct variational problems which are well-posed and for which the transport interpolations are solutions in some sense. We believe that these variational problems are interesting in their own right. In order to describe the solutions of the variational problem, we will rely on a dual approach based on the Hamilton-Jacobi equation, inspired from Fathi's approach to Mather theory, as detailed in Section 3 The solutions of the problems of Section 2, as well as the transport interpolations, are then described in Section 4 , which ends the proof of Theorem A.

\subsection{Case of an absolutely continuous measure $\mu_{0}$}

Additional conclusions concerning optimal transport can usually be obtained when the initial measure $\mu_{0}$ is absolutely continuous. For example a standard question is whether the optimal transport can be realized by an optimal mapping.

A transport map is a Borel map $\Psi: M \rightarrow M$ which satisfies $\Psi_{\sharp} \mu_{0}=\mu_{1}$. To any transport map $\Psi$ is naturally associated the transport plan $(\operatorname{Id} \times \Psi)_{\sharp} \mu_{0}$, called the induced transport plan. An optimal map is a transport map $\Psi: M \rightarrow M$ such that

$$
\int_{M} c_{T}(x, \Psi(x)) d \mu_{0} \leq \int_{M} c_{T}(x, F(x)) d \mu_{0}
$$

for any transport map $F$. It turns out that, under the assumption that $\mu_{0}$ has no atoms, a transport map is optimal if and only if the induced transport plan is an optimal transport plan (see [1, Theorem 2.1]). In other words, we have

$$
\inf _{\Psi} \int_{M} c(x, \Psi(x)) d \mu_{0}(x)=C\left(\mu_{0}, \mu_{1}\right),
$$

where the infimum is taken over the set of transport maps from $\mu_{0}$ to $\mu_{1}$. This is a general result which holds for any continuous cost $c$. It is a standard question, which turns out to be very hard for certain cost functions, whether the infimum above is reached, or in other words whether there exists an optimal transport plan which is induced from a transport map. Part of the result below is that this holds true in the case of the $\operatorname{cost} c_{0}^{T}$. The method we use to prove this is an elaboration on ideas due to Brenier [11] and developed for instance in [23] (see also [22]) and [15], which is certainly the closest to our needs.

Theorem B. Assume that $\mu_{0}$ is absolutely continuous with respect to the Lebesgue class on $M$. Then for each final measure $\mu_{T}$, there exists a unique interpolation $\mu_{t}, t \in[0, T]$, and each interpolating measure $\mu_{t}, t<T$, is absolutely continuous. In addition, there 
exists a family $\left.\left.\Psi_{0}^{t}: M \rightarrow M, t \in\right] 0, T\right]$, of Borel maps such that (Id $\left.\times \Psi_{0}^{t}\right)_{\sharp} \mu_{0}$ is the only optimal transfer plan in $\mathcal{K}\left(\mu_{0}, \mu_{t}\right)$ for the cost function $c_{0}^{t}$. Consequently, we have

$$
\int_{M} c_{0}^{t}\left(x, \Psi_{0}^{t}(x)\right) d \mu_{0}(x)=C_{0}^{t}\left(\mu_{0}, \mu_{t}\right), \quad 0<t \leq T .
$$

If $\mu_{T}$, instead of $\mu_{0}$, is assumed to be absolutely continuous, then there exists a unique interpolation, and each interpolating measure $\left.\left.\mu_{t}, t \in\right] 0, T\right]$, is absolutely continuous.

This theorem will be proved and commented in Section 5

\subsection{Mather theory}

Let us now assume that the Lagrangian function is defined for all times, $L \in C^{2}(T M \times$ $\mathbb{R}, \mathbb{R})$, and, in addition to the standing hypotheses, satisfies the periodicity condition

$$
L(x, v, t+1)=L(x, v, t)
$$

for all $(x, v, t) \in T M \times \mathbb{R}$. A Mather measure (see [30]) is a compactly supported probability measure $m_{0}$ on $T M$ which is invariant in the sense that $\left(\psi_{0}^{1}\right)_{\sharp} m_{0}=m_{0}$ and which minimizes the action

$$
A_{0}^{1}\left(m_{0}\right)=\int_{T M \times[0,1]} L\left(\psi_{0}^{t}(x, v), t\right) d m_{0} d t .
$$

The major discovery of [30] is that Mather measures are supported on the graph of a Lipschitz vector field. Let us denote by $\alpha$ the action of Mather measures - this number is the value at zero of the $\alpha$ function defined by Mather in [30]. Let us now explain how this theory of Mather is related to, and can be recovered from, the content of our paper.

Theorem C. We have

$$
\alpha=\min _{\mu} C_{0}^{1}(\mu, \mu)
$$

where the minimum is taken over the set of probability measures on M. The mapping $m_{0} \mapsto \pi_{\sharp} m_{0}$ is a bijection between the set of Mather measures $m_{0}$ and the set of probability measures $\mu$ on $M$ satisfying $C_{0}^{1}(\mu, \mu)=\alpha$. There exists a Lipschitz vector field $X_{0}$ on $M$ such that all the Mather measures are supported on the graph of $X_{0}$.

This theorem will be proved in Section 6, where the bijection between Mather measures and measures minimizing $C_{0}^{1}(\mu, \mu)$ will be specified.

\section{Direct variational problems}

We state two different variational problems whose solutions are the interpolated transports. We believe that these problems are interesting in their own right. They will also be used to prove Theorem A. 


\subsection{Measures}

This formulation parallels Mather's theory. It can also be related to the generalized curves of L. C. Young. Let $\mu_{0}$ and $\mu_{T}$ be two probability Borel measures on $M$. Let $m_{0} \in$ $\mathcal{B}_{1}(T M)$ be a Borel probability measure on the tangent bundle $T M$. We say that $m_{0}$ is an initial transport measure if the measure $\eta$ on $M \times M$ given by

$$
\eta=\left(\pi \times\left(\pi \circ \psi_{0}^{T}\right)\right)_{\sharp} m_{0}
$$

is a transport plan, where $\pi: T M \rightarrow M$ is the canonical projection. We denote by $\mathcal{I}\left(\mu_{0}, \mu_{T}\right)$ the set of initial transport measures. To an initial transport measure $m_{0}$, we associate the continuous family of measures

$$
m_{t}=\left(\psi_{0}^{t}\right)_{\sharp} m_{0}, \quad t \in[0, T],
$$

on $T M$, and the measure $m$ on $T M \times[0, T]$ given by

$$
m=m_{t} \otimes d t=\left(\left(\psi_{0}^{t}\right)_{\sharp} m_{0}\right) \otimes d t .
$$

Note that the linear mapping $m_{0} \mapsto m=\left(\left(\psi_{0}^{t}\right) \sharp m_{0}\right) \otimes d t$ is continuous from $\mathcal{B}(T M)$ to $\mathcal{B}(T M \times[0, T])$ endowed with the weak topology (see Appendix).

Lemma 4. The measure $m$ satisfies the relation

$$
\int_{T M \times[0, T]}\left(\partial_{t} f(x, t)+\partial_{x} f(x, t) \cdot v\right) d m(x, v, t)=\int_{M} f_{T} d \mu_{T}-\int_{M} f_{0} d \mu_{0}
$$

for each $f \in C^{1}(M \times[0, T], \mathbb{R})$, where $f_{t}$ denotes the function $x \mapsto f(x, t)$.

Proof. Setting $\tilde{f}(x, v, t)=f(x, t), g_{1}(x, v, t)=\partial_{t} f(x, t)=\partial_{t} \tilde{f}(x, v, t)$ and $g_{2}(x, v, t)$ $=\partial_{x} f(x, t) \cdot v$, we have

$$
\int_{T M \times[0, T]}\left(\partial_{t} f(x, t)+\partial_{x} f(x, t) \cdot v\right) d m(x, v, t)=\int_{0}^{T} \int_{T M}\left(g_{1}+g_{2}\right) \circ \psi_{0}^{t} d m_{0} d t
$$

Noticing that, in view of equation (1), we have

$$
\partial_{t}\left(\tilde{f} \circ \psi_{0}^{t}\right)=g_{1} \circ \psi_{0}^{t}+g_{2} \circ \psi_{0}^{t},
$$

we obtain

$$
\begin{aligned}
\int_{T M \times[0, T]}\left(\partial_{t} f(x, t)+\partial_{x} f(x, t) \cdot v\right) d m(x, v, t) & =\int_{T M}\left(\tilde{f} \circ \psi_{0}^{T}-\tilde{f}\right) d m_{0} \\
& =\int_{M} f_{T} d \mu_{T}-\int_{M} f_{0} d \mu_{0}
\end{aligned}
$$

as desired. 
Definition 5. A finite Borel measure $m$ on $T M \times[0, T]$ which satisfies (5) is called a transport measure. We denote by $\mathcal{M}\left(\mu_{0}, \mu_{T}\right)$ the set of transport measures. A transport measure which is induced from an initial measure $m_{0}$ is called an invariant transport measure. The action of the transport measure $m$ is defined by

$$
A(m)=\int_{T M \times[0, T]} L(x, v, t) d m \in \mathbb{R} \cup\{\infty\} .
$$

The action $A\left(m_{0}\right)$ of an initial transport measure is defined as the action of the associated transport measure $m$. We will also denote this action by $A_{0}^{T}\left(m_{0}\right)$ when we want to indicate the time interval. We have

$$
A_{0}^{T}\left(m_{0}\right)=\int_{T M \times[0, T]} L\left(\psi_{0}^{t}(x, v), t\right) d m_{0} d t .
$$

Notice that initial transport measures exist:

Proposition 6. The mapping $\left(\pi \times\left(\pi \circ \psi_{0}^{T}\right)\right)_{\sharp}: \mathcal{I}\left(\mu_{0}, \mu_{T}\right) \rightarrow \mathcal{K}\left(\mu_{0}, \mu_{T}\right)$ is surjective. In addition, for each transport plan $\eta$, there exists a compactly supported initial transport measure $m_{0}$ such that $\left(\pi \times\left(\pi \circ \psi_{0}^{T}\right)\right)_{\sharp} m_{0}=\eta$ and

$$
A\left(m_{0}\right)=\int_{M \times M} c_{0}^{T}(x, y) d \eta .
$$

Proof. By Proposition1, there exists a compact set $K \subset T M$ such that if $\gamma:[0, T] \rightarrow M$ is a minimizing extremal, then the lifting $(\gamma(t), \dot{\gamma}(t))$ is contained in $K$ for each $t \in$ $[0, T]$. We shall prove that, for each probability measure $\eta \in \mathcal{B}(M \times M)$, there exists a probability measure $m_{0} \in \mathcal{B}(K)$ such that $\left(\pi \times\left(\pi \circ \psi_{0}^{T}\right)\right)_{\sharp} m_{0}=\eta$ and

$$
A\left(m_{0}\right)=\int_{M \times M} c_{0}^{T}(x, y) d \eta
$$

Observing that

- the mappings $m_{0} \mapsto\left(\pi \times\left(\pi \circ \psi_{0}^{T}\right)\right)_{\sharp} m_{0}$ and $m_{0} \mapsto A\left(m_{0}\right)$ are linear and continuous on the space $\mathcal{B}_{1}(K)$ of probability measures supported on $K$,

- $\mathcal{B}_{1}(K)$ is compact for the weak topology, and the action $A$ is continuous on this set,

- the set of probability measures on $M \times M$ is the compact convex closure of the set of Dirac probability measures (probability measures supported in one point; see e.g. [9]. p. 73]),

it is enough to prove the result when $\eta$ is a Dirac probability measure (or equivalently when $\mu_{0}$ and $\mu_{T}$ are Dirac probability measures). Let $\eta$ be the Dirac probability measure supported at $\left(x_{0}, x_{1}\right) \in M \times M$. Let $\gamma:[0, T] \rightarrow M$ be a minimizing extremal with boundary conditions $\gamma(0)=x_{0}$ and $\gamma(T)=x_{1}$. In view of the choice of $K$, we have $(\gamma(0), \dot{\gamma}(0)) \in K$. Let $m_{0}$ be the Dirac probability measure supported at $(\gamma(0), \dot{\gamma}(0))$. It is straightforward that $m_{t}$ is then the Dirac measure supported at $(\gamma(t), \dot{\gamma}(t))$, so that

$$
A\left(m_{0}\right)=\int_{0}^{T} L d m_{t} d t=\int_{0}^{T} L(\gamma(t), \dot{\gamma}(t), t) d t=c_{0}^{T}\left(x_{0}, x_{1}\right)=\int_{M \times M} c_{0}^{T} d \eta
$$

and $\left(\pi \times\left(\pi \circ \psi_{0}^{T}\right)\right)_{\sharp} m_{0}=\eta$. 
Although we are going to build minimizers by other means, we believe the following result is worth mentioning.

Lemma 7. For each real number $a$, the set $\mathcal{M}^{a}\left(\mu_{0}, \mu_{T}\right)$ of transport measures $m$ which satisfy $A(m) \leq a$, as well as the set $\mathcal{I}^{a}\left(\mu_{0}, \mu_{T}\right)$ of initial transport measures $m_{0}$ which satisfy $A_{0}^{T}\left(m_{0}\right) \leq a$, are compact. As a consequence, there exist optimal initial transport measures, and optimal transport measures.

Proof. This is an easy application of the Prokhorov theorem (see Appendix).

Now that we have seen that the problem of finding optimal transport measures is wellposed, let us describe its solutions.

Theorem 1. We have

$$
C_{0}^{T}\left(\mu_{0}, \mu_{T}\right)=\min _{m \in \mathcal{M}\left(\mu_{0}, \mu_{T}\right)} A(m)=\min _{m_{0} \in \mathcal{I}\left(\mu_{0}, \mu_{T}\right)} A\left(m_{0}\right) .
$$

The mapping

$$
m_{0} \mapsto m=\left(\left(\psi_{0}^{t}\right)_{\sharp} m_{0}\right) \otimes d t
$$

between the set $\mathcal{O I}$ of optimal initial measures and the set $\mathcal{O M}$ of optimal transport measures is a bijection. There exists a bounded and locally Lipschitz vector field $X$ : $M \times] 0, T\left[\rightarrow T M\right.$ such that, for each optimal initial measure $m_{0} \in \mathcal{O} \mathcal{I}$, the measure $m_{t}=\left(\psi_{0}^{t}\right)_{\sharp} m_{0}$ is supported on the graph of $X_{t}$ for each $\left.t \in\right] 0, T[$.

The proof will be given in Section 4.3 . Let us just notice now that the inequalities

$$
C_{0}^{T}\left(\mu_{0}, \mu_{T}\right) \geq \min _{m_{0} \in \mathcal{I}\left(\mu_{0}, \mu_{T}\right)} A\left(m_{0}\right) \geq \min _{m \in \mathcal{M}\left(\mu_{0}, \mu_{T}\right)} A(m)
$$

hold in view of Proposition 6

\subsection{Currents}

This formulation finds its roots on one hand in the works of Benamou and Brenier [6] and then Brenier [12], and on the other hand in the work of Bangert [5]. Let $\Omega^{0}(M \times[0, T])$ be the set of continuous one-forms on $M \times[0, T]$, endowed with the uniform norm. We will often decompose forms $\omega \in \Omega^{0}(M \times[0, T])$ as

$$
\omega=\omega^{x}+\omega^{t} d t
$$

where $\omega^{x}$ is a time-dependent form on $M$ and $\omega^{t}$ is a continuous function on $M \times[0, T]$. To each continuous linear form $\chi$ on $\Omega^{0}(M \times[0, T])$, we associate its time component $\mu_{\chi}$, which is the measure on $M \times[0, T]$ defined by

$$
\int_{M \times[0, T]} f d \mu_{\chi}=\chi(f d t)
$$

for each continuous function $f$ on $M \times[0, T]$. A transport current between $\mu_{0}$ and $\mu_{T}$ is a continuous linear form $\chi$ on $\Omega^{0}(M \times[0, T])$ which satisfies the two conditions: 
1. The measure $\mu_{\chi}$ is non-negative (and bounded).

2. $d \chi=\mu_{T} \otimes \delta_{T}-\mu_{0} \otimes \delta_{0}$, which means that

$$
\chi(d f)=\int_{M} f_{T} d \mu_{T}-\int_{M} f_{0} d \mu_{0}
$$

for each smooth (or equivalently $C^{1}$ ) function $f: M \times[0, T] \rightarrow \mathbb{R}$.

We let $\mathcal{C}\left(\mu_{0}, \mu_{T}\right)$ denote the set of transport currents from $\mu_{0}$ to $\mu_{T}$. It is a closed convex subset of $\left[\Omega^{0}(M \times[0, T])\right]^{*}$. We will endow $\mathcal{C}\left(\mu_{0}, \mu_{T}\right)$ with the weak topology obtained as the restriction of the weak* topology of $\left[\Omega^{0}(M \times[0, T])\right]^{*}$. Transport currents should be thought of as vector fields whose components are measures, the last component being $\mu_{\chi}$.

If $Z$ is a bounded measurable vector field on $M \times[0, T]$, and if $v$ is a finite nonnegative measure on $M \times[0, T]$, we define the current $Z \wedge v$ by

$$
Z \wedge \nu(\omega):=\int_{M \times[0, T]} \omega(Z) d \nu
$$

Every transport current can be written in this way (see [21] or [24]). As a consequence, currents extend to linear forms on the set $\Omega_{\infty}(M \times[0, T])$ of bounded measurable oneforms. If $I$ is a Borel subset of $[0, T]$, it is therefore possible to define the restriction $\chi_{I}$ of the current $\chi$ to $I$ by the formula $\chi_{I}(\omega)=\chi\left(1_{I} \omega\right)$, where $1_{I}$ is the indicatrix of $I$.

Lemma 8. If $\chi$ is a transport current, then

$$
\tau_{\sharp} \mu_{\chi}=d t,
$$

where $\tau$ is the projection onto $[0, T]$ (see Appendix). As a consequence, there exists a measurable family $\left.\mu_{t}, t \in\right] 0, T\left[\right.$, of probability measures on $M$ such that $\mu_{\chi}=\mu_{t} \otimes d t$ (see Appendix). There exists a set I $\subset$ ]0, T[ of full measure such that

$$
\int_{M} f_{t} d \mu_{t}=\int_{M} f_{0} d \mu_{0}+\chi_{[0, t[}(d f)
$$

for each $C^{1}$ function $f: M \times[0, T] \rightarrow M$ and each $t \in I$.

Proof. Let $g:[0, T] \rightarrow \mathbb{R}$ be a continuous function. Setting $G(t)=\int_{0}^{t} g(s) d s$, we observe that

$\int_{M \times[0, T]} g d \mu_{\chi}=\chi(d G)=\int_{M} G_{T} d \mu_{T}-\int_{M} G_{0} d \mu_{0}=G(T)-G(0)=\int_{0}^{T} g(s) d s$.

This implies that $\tau_{\sharp} \mu_{\chi}=d t$. As a consequence, the measure $\mu_{\chi}$ can be desintegrated as $\mu_{\chi}=\mu_{t} \otimes d t$. We claim that, for each $C^{1}$ function $f: M \times[0, T] \rightarrow M$, the relation (6) holds for almost every $t$. Since the space $C^{1}(M \times[0, T], \mathbb{R})$ is separable, the claim implies the existence of a set $I \subset$ ]0, $T$ [ of full Lebesgue measure such that $(6)$ holds for all $t \in I$ 
and all $f \in C^{1}(M \times[0, T], \mathbb{R})$. In order to prove the claim, fix $f$ in $C^{1}(M \times[0, T], \mathbb{R})$. For each $g \in C^{1}([0, T], \mathbb{R})$, we have

$$
\chi(d(g f))=\chi\left(g^{\prime} f d t\right)+\chi(g d f),
$$

hence

$$
g(T) \int_{M} f_{T} d \mu_{T}-g(0) \int_{M} f_{0} d \mu_{0}=\int_{0}^{T} g^{\prime}(t) \int_{M} f_{t} d \mu_{t} d t+\chi(g d f) .
$$

By applying this relation to a sequence of $C^{1}$ functions $g$ approximating $1_{[0, t[}$, we get, in the limit,

$$
-\int_{M} f_{0} d \mu_{0}=-\int_{M} f_{t} d \mu_{t}+\chi_{[0, t[}(d f)
$$

at every Lebesgue point of the function $t \mapsto \int_{M} f_{t} d \mu_{t}$.

If $\mu_{0}=\mu_{T}$, an easy example of a transport current is given by $\chi(\omega)=\int_{M} \int_{0}^{T} \omega^{t} d t d \mu_{0}$. Here are some more interesting examples.

Regular transport currents. The transport current $\chi$ is called regular if there exists a bounded measurable section $X$ of the projection $T M \times[0, T] \rightarrow M \times[0, T]$, and a nonnegative measure $\mu$ on $M \times[0, T]$ such that $\chi=(X, 1) \wedge \mu$. The time component of the current $(X, 1) \wedge \mu$ is $\mu$. In addition, if $(X, 1) \wedge \mu=\left(X^{\prime}, 1\right) \wedge \mu$ for two vector fields $X$ and $X^{\prime}$, then $X$ and $X^{\prime}$ agree $\mu$-almost everywhere.

The current $\chi=(X, 1) \wedge \mu$, with $X$ bounded, is a regular transport current if and only if there exists a (unique) continuous family $\mu_{t} \in \mathcal{B}_{1}(M), t \in[0, T]$ (where $\mu_{0}$ and $\mu_{T}$ are the transported measures), such that $\mu_{\chi}=\mu_{t} \otimes d t$ and such that the transport equation

$$
\partial_{t} \mu_{t}+\partial_{x} \cdot\left(X \mu_{t}\right)=0
$$

holds in the sense of distributions on $M \times] 0, T[$. The relation

$$
\int_{M} f_{t} d \mu_{t}-\int_{M} f_{s} d \mu_{s}=\chi_{[s, t[}(d f)
$$

then holds for each $C^{1}$ function $f$ and any $s \leq t$ in $[0, T]$.

In order to prove that the family $\mu_{t}$ can be chosen continuous, pick a function $f \in$ $C^{1}(M, \mathbb{R})$ and notice that the equation

$$
\int_{M} f d \mu_{t}-\int_{M} f d \mu_{s}=\chi_{[s, t[}(d f)=\int_{s}^{t} \int_{M} d f \cdot X_{\sigma} d \mu_{\sigma} d \sigma
$$

holds for all $s \leq t$ in a subset $I \subset[0, T]$ of full measure. Note that this relation also holds if $s=0$ and $t \in I$ and if $s \in I$ and $t=T$. Since the function $\sigma \mapsto \int_{M} d f \cdot X_{\sigma} d \mu_{\sigma}$ is bounded, we conclude that the function $t \mapsto \int_{M} f d \mu_{t}$ is Lipschitz on $I \cup\{0, T\}$ for each $f \in C^{1}(M, \mathbb{R})$, with a Lipschitz constant which depends only on $\|d f\|_{\infty} \cdot\|X\|_{\infty}$. The family $\mu_{t}$ is then Lipschitz on $I \cup\{0, T\}$ for the 1-Wasserstein distance on probability 
measures (see [35, 16, 3] for example), the Lipschitz constant depending only on $\|X\|_{\infty}$. It suffices to remember that, on the compact manifold $M$, the 1 -Wasserstein distance on probabilities is topologically equivalent to the weak topology (see for example [37, (48.5)], or [35]).

Smooth transport currents. A regular transport current is said to be smooth if it can be written in the form $(X, 1) \wedge \lambda$ with a bounded vector field $X$ smooth on $M \times] 0, T$ [ and a measure $\lambda$ that has a positive smooth density with respect to the Lebesgue class in any chart in $M \times] 0, T$ [. Every transport current in $\mathcal{C}\left(\mu_{0}, \mu_{T}\right)$ can be approximated by smooth transport currents, but we shall not use such approximations.

Lipschitz regular transport currents. A regular transport current is called Lipschitz regular if it can be written in the form $(X, 1) \wedge \mu$ with a vector field $X$ which is bounded and locally Lipschitz on $M \times] 0, T$ [. Smooth currents are Lipschitz regular. Lipschitz regular transport currents have a remarkable structure:

If $\chi=(X, 1) \wedge \mu$ is a Lipschitz regular transport current with $X$ bounded and locally Lipschitz on $M \times] 0, T[$, then

$$
\left(\Psi_{s}^{t}\right)_{\sharp} \mu_{s}=\mu_{t}
$$

where $\left.\Psi_{s}^{t},(s, t) \in\right] 0, T\left[{ }^{2}\right.$, denotes the flow of the Lipschitz vector field $X$ from time $s$ to time $t$, and $\mu_{t}$ is the continuous family of probability measures such that $\mu_{\chi}=\mu_{t} \otimes d t$.

This statement follows from standard representation results for solutions of the transport equation (see for example [2] or [3]).

Transport current induced from a transport measure. To a transport measure $m$, we associate the transport current $\chi_{m}$ defined by

$$
\chi_{m}(\omega)=\int_{T M \times[0, T]}\left(\omega^{x}(x, t) \cdot v+\omega^{t}(x, t)\right) d m(x, v, t)
$$

where the form $\omega$ is decomposed as $\omega=\omega^{x}+\omega^{t} d t$. Note that the time component of the current $\chi_{m}$ is $\pi_{\sharp} m$. We will see in Lemma 11 that

$$
A\left(\chi_{m}\right) \leq A(m)
$$

with the following definition of the action $A(\chi)$ of a current, with equality if $m$ is concentrated on the graph of any bounded vector field $M \times[0, T] \rightarrow T M$.

Lemma 9. For each transport current $\chi$, the numbers

$$
\begin{aligned}
& A_{1}(\chi)=\sup _{\omega \in \Omega^{0}}\left(\chi\left(\omega^{x}, 0\right)-\int_{M \times[0, T]} H\left(x, \omega^{x}(x, t), t\right) d \mu_{\chi}\right), \\
& A_{2}(\chi)=\sup _{\omega \in \Omega^{0}}\left(\chi(\omega)-\int_{M \times[0, T]}\left(H\left(x, \omega^{x}(x, t), t\right)+\omega^{t}\right) d \mu_{\chi}\right), \\
& A_{3}(\chi)=\sup _{\omega \in \Omega^{0}}\left(\chi(\omega)-T \sup _{(x, t) \in M \times[0, T]}\left(H\left(x, \omega^{x}(x, t), t\right)+\omega^{t}\right)\right),
\end{aligned}
$$




$$
\begin{aligned}
& A_{4}(\chi)=\sup _{\omega \in \Omega^{0}, \omega^{t}+H\left(x, \omega^{x}, t\right) \leq 0} \chi(\omega), \\
& A_{5}(\chi)=\sup _{\omega \in \Omega^{0}, \omega^{t}+H\left(x, \omega^{x}, t\right) \equiv 0} \chi(\omega) .
\end{aligned}
$$

are equal. In addition the numbers $A_{i}^{\infty}(\chi)$ obtained by replacing in the above suprema the set $\Omega^{0}$ of continuous forms by the set $\Omega_{\infty}$ of bounded measurable forms also have the same value.

The last remark in the statement has been added in the last version of the paper and is inspired by [14].

Proof. It is straightforward that $A_{1}=A_{2}$ : this just amounts to simplifying the term $\int \omega^{t} d \mu_{\chi}$. Since $\mu_{\chi}$ is a non-negative measure which satisfies $\int_{M \times[0, T]} 1 d \mu_{\chi}=T$, we have

$$
\int_{M \times[0, T]}\left(H\left(x, \omega^{x}(x, t), t\right)+\omega^{t}\right) d \mu_{\chi} \leq T \sup _{(x, t) \in M \times[0, T]}\left(H\left(x, \omega^{x}(x, t), t\right)+\omega^{t}\right)
$$

so that $A_{3}(\chi) \leq A_{2}(\chi)$. In addition, we obviously have $A_{5}(\chi) \leq A_{4}(\chi) \leq A_{3}(\chi)$. Now notice that, in $A_{2}$, the quantity

$$
\chi(\omega)-\int_{M \times[0, T]}\left(H\left(x, \omega^{x}(x, t), t\right)+\omega^{t}\right) d \mu_{\chi}
$$

does not depend on $\omega^{t}$. Consider the form $\tilde{\omega}=\left(\omega^{x},-H\left(x, \omega^{x}, t\right)\right)$, which satisfies the equality $H\left(x, \tilde{\omega}^{x}, t\right)+\tilde{\omega}^{t} \equiv 0$. We get, for each form $\omega$,

$$
\chi\left(\omega^{x}, 0\right)-\int_{M \times[0, T]} H\left(x, \omega^{x}(x, t), t\right) d \mu_{\chi}=\chi(\tilde{\omega}) \leq A_{5}(\chi) .
$$

Hence $A_{1}(\chi) \leq A_{5}(\chi)$. Exactly the same proof shows that the numbers $A_{i}^{\infty}(\chi)$ are equal. In order to end the proof, it is enough to check that $A_{2}(\chi)=A_{2}^{\infty}(\chi)$. Writing the current $\chi$ in the form $Z \wedge v$ with a bounded vector field $Z$ and a measure $v \in \mathcal{B}_{+}(M \times[0, T])$, we have

$$
A_{2}(\chi)=\sup _{\omega \in \Omega^{0}}\left(\int_{M \times[0, T]} \omega(Z) d \nu-\int_{M \times[0, T]}\left(H\left(x, \omega^{x}(x, t), t\right)+\omega^{t}\right) d \mu_{\chi}\right)
$$

and

$$
A_{2}^{\infty}(\chi)=\sup _{\omega \in \Omega_{\infty}}\left(\int_{M \times[0, T]} \omega(Z) d \nu-\int_{M \times[0, T]}\left(H\left(x, \omega^{x}(x, t), t\right)+\omega^{t}\right) d \mu_{\chi}\right) .
$$

The desired result follows by density of continuous functions in $L^{1}\left(v+\mu_{\chi}\right)$.

Definition 10. We denote by $A(\chi)$ the common value of the numbers $A_{i}(\chi)$ and call it the action of the transport current $\chi$. 
The existence of currents of finite action follows from

Lemma 11. We have

$$
A(\chi)=\int_{M \times[0, T]} L(x, X(x, t), t) d \mu
$$

for each regular current $\chi=(X, 1) \wedge \mu$. If $m$ is a transport measure, and if $\chi_{m}$ is the associated transport current, then $A\left(\chi_{m}\right) \leq A(m)$, with equality if $m$ is supported on the graph of a bounded Borel vector field. As a consequence,

$$
C_{0}^{T}\left(\mu_{0}, \mu_{T}\right) \geq \min _{m_{0} \in \mathcal{I}\left(\mu_{0}, \mu_{T}\right)} A\left(m_{0}\right) \geq \min _{m \in \mathcal{M}\left(\mu_{0}, \mu_{T}\right)} A(m) \geq \min _{\chi \in \mathcal{C}\left(\mu_{0}, \mu_{T}\right)} A(\chi) .
$$

Proof. For each bounded measurable form $\omega$, we have

$$
\int_{M \times[0, T]}\left(\omega^{x}(X)-H\left(x, \omega^{x}(x, t), t\right)\right) d \mu \leq \int_{M \times[0, T]} L(x, X(x, t), t) d \mu,
$$

so that

$$
A((X, 1) \wedge \mu) \leq \int_{M \times[0, T]} L(x, X(x, t), t) d \mu .
$$

On the other hand, taking the form $\omega_{0}^{x}(x, t)=\partial_{v} L(x, X(x, t), t)$ we obtain the pointwise equality

$$
L(x, X(x, t), t)=\omega_{0}^{x}(X)-H\left(x, \omega_{0}^{x}(x, t), t\right)
$$

and by integration

$$
\begin{aligned}
\int_{M \times[0, T]} L(x, X(x, t), t) d \mu & =\int_{M \times[0, T]}\left(\omega_{0}^{x}(X)-H\left(x, \omega_{0}^{x}(x, t), t\right)\right) d \mu \\
& \leq A((X, 1) \wedge \mu) .
\end{aligned}
$$

This ends the proof of the equality of the two forms of the action of regular currents. Now if $\chi_{m}$ is the current associated to a transport measure $m$, then, for each bounded form $\omega \in \Omega^{0}(M \times[0, T])$, we have

$$
\begin{aligned}
\chi_{m}(\omega)-\int_{M \times[0, T]}\left(\omega^{t}(x, t)+H(x,\right. & \left.\left.\omega^{x}(x, t), t\right)\right) d \mu_{\chi} \\
& =\int_{T M \times[0, T]}\left(\omega^{x}(v)-H\left(x, \omega^{x}(x, t), t\right)\right) d m
\end{aligned}
$$

by definition of $\chi_{m}$, so that

$$
A\left(\chi_{m}\right) \leq \int_{T M \times[0, T]} L(x, v, t) d m=A(m)
$$

by the Legendre inequality. In addition, if there exists a bounded measurable vector field $X: M \times[0, T] \rightarrow T M$ such that the graph of $X \times \tau$ supports $m$, then we can consider the form $\omega_{0}^{x}$ associated to $X$ as above, and we get the equality for this form.

Although we are going to provide explicitly a minimum of $A$, we believe the following lemma is worth mentioning. 
Lemma 12. The functional $A: \mathcal{C}\left(\mu_{0}, \mu_{T}\right) \rightarrow \mathbb{R} \cup\{+\infty\}$ is convex and lower semicontinuous, both for the strong and weak $k^{*}$ topologies on $\left[\Omega^{0}(M \times[0, T])\right]^{*}$. Moreover it is coercive with respect to the strong topology and hence it has a minimum.

Proof. First note that $A(\chi)<\infty$ if $\chi$ is the transport current corresponding to an initial transport measure in $\mathcal{M}\left(\mu_{0}, \mu_{T}\right)$ arising from a transport plan. Define the continuous convex function $\mathbb{H}_{T}: \Omega^{0}(M \times[0, T]) \rightarrow \mathbb{R}$ by

$$
\mathbb{H}_{T}(\omega)=T \sup _{(x, t) \in M \times[0, T]}\left(H\left(x, \omega^{x}(x, t), t\right)+\omega^{t}\right) .
$$

Then the action is the restriction to $\mathcal{C}\left(\mu_{0}, \mu_{T}\right)$ of the Fenchel conjugate $A=\mathbb{H}^{*}$ : $\left[\Omega^{0}(M \times[0, T])\right]^{*} \rightarrow \mathbb{R} \cup\{+\infty\}$. In other words, $A$ is the supremum over $\omega$ of the family of affine functionals

$$
\chi \mapsto \chi(\omega)-\mathbb{H}_{T}(\omega)
$$

that are continuous both for the strong and weak* topologies. Hence $A$ is convex and lower semicontinuous for both topologies. Since

$$
A(\chi) \geq \sup _{\|\omega\| \leq 1} \chi(\omega)-\sup _{\|\omega\| \leq 1} \mathbb{H}_{T}(\omega)
$$

$A$ is coercive. The existence of a minimizer is standard: any minimizing sequence $\left(\chi_{n}\right)$ is bounded (thanks to coercivity) and has a weak* convergent subsequence (because $\Omega^{0}(M \times[0, T])$ is a separable Banach space). By lower semicontinuity, its weak* limit is a minimizer. Note that $\mathcal{C}\left(\mu_{0}, \mu_{T}\right)$ is weak* closed.

Theorem 2. We have

$$
C_{0}^{T}\left(\mu_{0}, \mu_{T}\right)=\min _{\chi \in \mathcal{C}\left(\mu_{0}, \mu_{T}\right)} A(\chi)
$$

where the minimum is taken over all transport currents from $\mu_{0}$ to $\mu_{T}$. Every optimal transport current is Lipschitz regular. Let $\chi=(X, 1) \wedge \mu$ be an optimal transport current, with $X$ locally Lipschitz on $M \times] 0, T\left[\right.$. The measure $m=(X \times \tau)_{\sharp} \mu \in \mathcal{B}_{+}(T M \times] 0, T[)$ is an optimal transport measure, and $\chi$ is the transport current induced from $m$. Here $\tau: T M \times[0, T] \rightarrow[0, T]$ is the projection on the second factor (see Appendix). We have

$$
C_{0}^{T}\left(\mu_{0}, \mu_{T}\right)=A(m)=A(\chi)=\int_{M \times[0, T]} L(x, X(x, t), t) d \mu_{\chi} .
$$

This result will be proved in 4.1 after establishing some essential results on the dual approach.

\section{Hamilton-Jacobi equation}

Most of the results stated so far can be proved by direct approaches using Mather's shortening lemma, which in a sense is an improvement on the initial observation of Monge (see [30] and [5]). We shall however base our proofs on the use of the Hamilton-Jacobi equation, in the spirit of Fathi's [19] approach to Mather theory, which should be associated to Kantorovich's dual approach to the transportation problem. 


\subsection{Viscosity solutions and semiconcave functions}

It is certainly useful to recall the main properties of viscosity solutions in connection with semiconcave functions. We will not give proofs, and instead refer to [19], [20], [13], as well as the appendix in [7]. We will consider the Hamilton-Jacobi equation

$$
\partial_{t} u+H\left(x, \partial_{x} u, t\right)=0 .
$$

The function $u: M \times[0, T] \rightarrow M$ is called $K$-semiconcave if, for each chart $\theta \in \Theta$ (see Appendix), the function

$$
(x, t) \mapsto u(\theta(x), t)-K\left(\|x\|^{2}+t^{2}\right)
$$

is concave on $B_{3} \times[0, T]$. The function $u$ is called semiconcave if it is $K$-semiconcave for some $K$. A function $u: M \times] 0, T[\rightarrow M$ is called locally semiconcave if it is semiconcave on each $M \times[s, t]$, for $0<s<t<T$. The following regularity result follows from Fathi's work [19] (see also [7]).

Proposition 13. Let $u_{1}$ and $u_{2}$ be two $K$-semiconcave functions. Let $A$ be the set of minima of the function $u_{1}+u_{2}$. Then the functions $u_{1}$ and $u_{2}$ are differentiable on $A$, and $d u_{1}(x, t)+d u_{2}(x, t)=0$ at each point of $(x, t) \in A$. In addition, the mapping $d u_{1}: M \times[0, T] \rightarrow T^{*} M$ is $C K$-Lipschitz continuous on A, where $C$ is a universal constant.

Definition 14. We say that $u: M \times] s, t[\rightarrow \mathbb{R}$ is a viscosity solution of $(H J)$ if

$$
u(x, \sigma)=\min _{y \in M}\left(u(y, \zeta)+c_{\zeta}^{\sigma}(y, x)\right) \quad \text { for all } x \in M \text { and } s<\zeta<\sigma<t .
$$

We say that $\breve{u}: M \times] s, t[\rightarrow \mathbb{R}$ is a backward viscosity solution of $(H J)$ if

$$
\breve{u}(x, \sigma)=\max _{y \in M}\left(\breve{u}(y, \zeta)-c_{\sigma}^{\zeta}(x, y)\right) \quad \text { for all } x \in M \text { and } s<\sigma<\zeta<t .
$$

We say that $v: M \times] s, t[\rightarrow \mathbb{R}$ is a viscosity subsolution of $(H J)$ if

$$
v(x, \sigma) \leq v(y, \zeta)+c_{\zeta}^{\sigma}(y, x) \quad \text { for all } x, y \in M \text { and } s<\zeta<\sigma<t .
$$

Finally, we say that $v: M \times[s, t] \rightarrow \mathbb{R}$ is a continuous viscosity solution (subsolution, backward solution) of ( $H J)$ if it is continuous on $M \times[s, t]$ and if $\left.v\right|_{M \times] s, t[}$ is a viscosity solution of $(H J)$ (subsolution, backward solution).

Notice that both viscosity solutions and backward viscosity solutions are viscosity subsolutions. That these definitions are equivalent in our setting to the usual ones is studied in the references listed above, but is not useful for our discussion. The only fact which will be used is that, for a $C^{1}$ function $\left.u: M \times\right] s, t[\rightarrow \mathbb{R}$, being a viscosity solution (or a backward viscosity solution) is equivalent to being a pointwise solution of $(H J)$, and being a viscosity subsolution is equivalent to satisfying the pointwise inequality $\partial_{t} u+H\left(x, \partial_{x} u, t\right) \leq 0$. 
Differentiability of viscosity solutions. Let $u \in C(M \times[0, T[, \mathbb{R})$ be a viscosity solution of $(H J)$ (on the interval ]0, $T[$ ). We have the expression

$$
u(x, t)=\min _{\gamma}\left(u(\gamma(0), 0)+\int_{0}^{t} L(\gamma(\sigma), \dot{\gamma}(\sigma), \sigma) d \sigma\right)
$$

where the minimum is taken over the set of curves $\gamma \in C^{2}([s, t], M)$ which satisfy the final condition $\gamma(t)=x$. Denote by $\Gamma(x, t)$ the set of minimizing curves in this expression, which are obviously minimizing extremals of $L$. We say that $p \in T_{x}^{*} M$ is a proximal superdifferential of a function $u: M \rightarrow \mathbb{R}$ at a point $x$ if there exists a smooth function $f: M \rightarrow \mathbb{R}$ such that $f-u$ has a minimum at $x$ and $d_{x} f=p$.

Proposition 15. Fix $(x, t) \in M \times] 0, T$ [. The function $u_{t}$ is differentiable at $x$ if and only if the set $\Gamma(x, t)$ contains a single element $\gamma$, and then $\partial_{x} u(x, t)=\partial_{v} L(x, \dot{\gamma}(t), t)$.

For all $(x, t) \in M \times] 0, T\left[\right.$ and $\gamma \in \Gamma(x, t)$, set $p(s)=\partial_{v} L(\gamma(s), \dot{\gamma}(s), s)$. Then $p(0)$ is a proximal subdifferential of $u_{0}$ at $\gamma(0)$, and $p(t)$ is a proximal superdifferential of $u_{t}$ at $x$.

We finish with an important statement on regularity of viscosity solutions:

Proposition 16. For each continuous function $u_{0}: M \rightarrow \mathbb{R}$, the viscosity solution

$$
u(x, t):=\min _{y \in M}\left(u_{0}(y)+c_{0}^{t}(y, x)\right)
$$

is locally semiconcave on ]0, $T$ ]. If in addition the initial condition $u_{0}$ is Lipschitz, then $u$ is Lipschitz on $[0, T]$.

For each continuous function $u_{T}: M \rightarrow \mathbb{R}$, the viscosity solution

$$
\breve{u}(x, t):=\max _{y \in M}\left(u_{T}(y)-c_{t}^{T}(x, y)\right)
$$

is locally semiconvex on [0, T[. If in addition the final condition $u_{T}$ is Lipschitz, then $u$ is Lipschitz on $[0, T]$.

Proof. The part concerning semiconcavity of $u$ is proved in [13], for example. It implies that $u$ is locally Lipschitz on ]0,T], hence differentiable almost everywhere. In addition, at each point of differentiability of $u$, we have $\partial_{t} u+H\left(x, \partial_{x} u, t\right)=0$ and $\partial_{x} u(x, t)=$ $p(t)=\partial_{v} L(x, \dot{\gamma}(t), t)$, where $\gamma:[0, t] \rightarrow M$ is the only curve in $\Gamma(x, t)$. In order to prove that $u$ is Lipschitz, it is enough to prove that there exists a uniform bound on $|p(t)|$. It is known (see Proposition 15p that $p(0):=\partial_{v} L(\gamma(0), \dot{\gamma}(0), 0)$ is a proximal subdifferential of $u_{0}$ at $\gamma(0)$. If $u_{0}$ is Lipschitz, its subdifferentials are bounded: there exists a constant $K$ such that $|p(0)| \leq K$. By completeness, there exists a constant $K^{\prime}$, which depends only on the Lipschitz constant of $u_{0}$, such that $|p(s)| \leq K^{\prime}$ for all $s \in$ $[0, t]$. This proves that $u$ is Lipschitz. The statements concerning $\breve{u}$ are proved in a similar way. 
3.2. Viscosity solutions and optimal Kantorovich pairs

Given an optimal Kantorovich pair $\left(\phi_{0}, \phi_{1}\right)$, we define the viscosity solution

$$
u(x, t):=\min _{y \in M}\left(\phi_{0}(x)+c_{0}^{t}(y, x)\right)
$$

and the backward viscosity solution

$$
\breve{u}(x, t):=\max _{y \in M}\left(\phi_{1}(y)-c_{t}^{T}(x, y)\right)
$$

which satisfy $u_{0}=\breve{u}_{0}=\phi_{0}$, and $u_{T}=\breve{u}_{T}=\phi_{1}$. Note that both $\phi_{1}$ and $-\phi_{0}$ are semiconcave, hence Lipschitz, $u$ is Lipschitz and locally semiconcave on ]0,T], and $\breve{u}$ is Lipschitz and locally semiconvex on $[0, T[$.

Proposition 17. We have

$$
C_{0}^{T}\left(\mu_{0}, \mu_{T}\right)=\max _{u}\left(\int_{M} u_{T} d \mu_{T}-\int_{M} u_{0} d \mu_{0}\right),
$$

where the minimum is taken over the set of continuous viscosity solutions $u: M \times$ $[0, T] \rightarrow \mathbb{R}$ of the Hamilton-Jacobi equation $(H J)$. The same conclusion holds if the maximum is taken over the set of continuous backward viscosity solutions, or over the set of continuous viscosity subsolutions of $(H J)$.

Proof. If $u(x, t)$ is a continuous viscosity subsolution of $(H J)$, then it satisfies

$$
u_{T}(x)-u_{0}(y) \leq c_{0}^{T}(y, x)
$$

for each $x$ and $y \in M$, and so, by Kantorovich duality,

$$
\int_{M} u_{T} d \mu_{T}-\int_{M} u_{0} d \mu_{0} \leq C_{0}^{T}\left(\mu_{0}, \mu_{T}\right)
$$

The converse inequality is obtained by using the functions $u$ and $\breve{u}$.

Definition 18. If $\left(\phi_{0}, \phi_{1}\right)$ is an optimal Kantorovich pair, then we denote by $\mathcal{F}\left(\phi_{0}, \phi_{1}\right) \subset$ $C^{2}([0, T], M)$ the set of curves $\gamma(t)$ such that

$$
\phi_{1}(\gamma(T))=\phi_{0}(\gamma(0))+\int_{0}^{T} L(\gamma(t), \dot{\gamma}(t), t) d t .
$$

We denote by $\left.\mathcal{T}\left(\phi_{0}, \phi_{1}\right) \subset M \times\right] 0, T[$ the set

$$
\mathcal{T}\left(\phi_{0}, \phi_{1}\right)=\{(\gamma(t), t): t \in] 0, T\left[, \gamma \in \mathcal{F}\left(\phi_{0}, \phi_{1}\right)\right\}
$$

and by $\left.\tilde{\mathcal{T}}\left(\phi_{0}, \phi_{1}\right) \subset T M \times\right] 0, T[$ the set

$$
\tilde{\mathcal{T}}\left(\phi_{0}, \phi_{1}\right)=\{(\gamma(t), \dot{\gamma}(t), t): t \in] 0, T\left[, \gamma \in \mathcal{F}\left(\phi_{0}, \phi_{1}\right)\right\},
$$

which is obviously invariant under the Euler-Lagrange flow. 
Proposition 19. Let $\left(\phi_{0}, \phi_{1}\right)$ be an optimal Kantorovich pair, and let $u$ and $\breve{u}$ be the associated viscosity and backward viscosity solutions.

1. We have $\breve{u} \leq u$, and

$$
\mathcal{T}\left(\phi_{0}, \phi_{1}\right)=\{(x, t) \in M \times] 0, T[: u(x, t)=\breve{u}(x, t)\} .
$$

2. At each point $(x, t) \in \mathcal{T}\left(\phi_{0}, \phi_{1}\right)$, the functions $u$ and $\breve{u}$ are differentiable, and satisfy $d u(x, t)=d \breve{u}(x, t)$. In addition, the mapping $(x, t) \mapsto d u(x, t)$ is locally Lipschitz on $\mathcal{T}\left(\phi_{0}, \phi_{1}\right)$.

3. If $\gamma(t) \in \mathcal{F}\left(\phi_{0}, \phi_{1}\right)$, then $\partial_{x} u(\gamma(t), t)=\partial_{v} L(\gamma(t), \dot{\gamma}(t), t)$. As a consequence, the set

$\mathcal{T}^{*}\left(\phi_{0}, \phi_{1}\right):=\left\{(x, p, t) \in T^{*} M \times\right] 0, T\left[:(x, t) \in \mathcal{T}\right.$ and $\left.p=\partial_{x} u(x, t)=\partial_{x} \breve{u}(x, t)\right\}$

is invariant under the Hamiltonian flow, and the restriction to $\tilde{\mathcal{T}}\left(\phi_{0}, \phi_{1}\right)$ of the projection $\pi$ is a bi-locally-Lipschitz homeomorphism onto its image $\mathcal{T}\left(\phi_{0}, \phi_{1}\right)$.

Proof. Fix $(x, t) \in M \times] 0, T\left[\right.$. There exist $y, z \in M$ such that $u(x, t)=\phi_{0}(y)+c_{0}^{t}(y, x)$ and $\breve{u}(x, t)=\phi_{1}(z)-c_{t}^{T}(x, z)$, so that

$$
\begin{aligned}
u(x, t)-\breve{u}(x, t) & =\phi_{0}(y)-\phi_{1}(z)+c_{0}^{t}(y, x)+c_{t}^{T}(x, z) \\
& \geq c_{0}^{T}(y, z)-\left(\phi_{1}(z)-\phi_{0}(y)\right) \geq 0 .
\end{aligned}
$$

In case of equality, we must have $c_{0}^{T}(y, z)=c_{0}^{t}(y, x)+c_{t}^{T}(x, z)$. Let $\gamma_{1} \in C^{2}([0, t], M)$ satisfy $\gamma_{1}(0)=y, \gamma_{1}(t)=x$ and $\int_{0}^{t} L\left(\gamma_{1}(s), \dot{\gamma}_{1}(s), s\right) d s=c_{0}^{t}(y, x)$, and let $\gamma_{2} \in$ $C^{2}([t, T], M)$ satisfy $\gamma_{2}(t)=x, \gamma_{2}(T)=z$ and $\int_{0}^{t} L\left(\gamma_{2}(s), \dot{\gamma}_{2}(s), s\right) d s=c_{t}^{T}(x, z)$. The curve $\gamma:[0, T] \rightarrow M$ obtained by pasting $\gamma_{1}$ and $\gamma_{2}$ clearly satisfies the equality $\int_{0}^{T} L(\gamma(s), \dot{\gamma}(s), s) d s=c_{0}^{T}(y, z)$, it is thus a $C^{2}$ minimizer, and belongs to $\mathcal{F}\left(\phi_{0}, \phi_{1}\right)$. As a consequence, we have $(x, t) \in \mathcal{T}\left(\phi_{0}, \phi_{1}\right)$.

Conversely, we have:

Lemma 20. If $v$ is a viscosity subsolution of $(H J)$ satisfying $v_{0}=\phi_{0}$ and $v_{T}=\phi_{1}$, then $\breve{u} \leq v \leq u$. If $(x, t) \in \mathcal{T}\left(\phi_{0}, \phi_{1}\right)$, then $v(x, t)=u(x, t)$.

Proof. The inequality $\breve{u} \leq v \leq u$ is easy. For example, for a given point $(x, t)$ there exists $y$ in $M$ such that $u(x, t)=\phi_{0}(y)+c_{0}^{t}(y, x)$, and for this value of $y$, we have $v(x, t) \leq$ $\phi_{0}(y)+c_{0}^{t}(y, x)$, hence $v(x, t) \leq u(x, t)$. The proof that $\breve{u} \leq v$ is similar. In order to prove the second part of the lemma, it is enough to prove that $v(\gamma(t), t)=u(\gamma(t), t)$ for each curve $\gamma \in \mathcal{F}\left(\phi_{0}, \phi_{1}\right)$. Since $v$ is a subsolution, we have

$$
v(\gamma(T), T) \leq v(\gamma(t), t)+c_{t}^{T}(\gamma(t), \gamma(T)) .
$$

On the other hand,

$$
v(\gamma(t), t) \leq u(\gamma(t), t) \leq u(\gamma(0), 0)+c_{0}^{t}(\gamma(0), \gamma(t)) .
$$


As a consequence,

$$
\begin{aligned}
\phi_{1}(\gamma(T)) & =v(\gamma(T), T) \leq u(\gamma(0), 0)+c_{0}^{t}(\gamma(0), \gamma(t))+c_{t}^{T}(\gamma(t), \gamma(T)) \\
& \leq \phi_{0}(\gamma(0))+c_{0}^{T}(\gamma(0), \gamma(T)),
\end{aligned}
$$

which is an equality because $\gamma \in \mathcal{F}\left(\phi_{0}, \phi_{1}\right)$. Hence all the inequalities involved are equalities, and we have $v(\gamma(t), t)=u(\gamma(t), t)$.

The end of the proof of the proposition is straightforward. Point 2 follows from Proposition 13 applied to the locally semiconcave functions $u$ and $-\breve{u}$. Point 3 follows from Proposition 15 .

\subsection{Optimal $C^{1}$ subsolution}

The following result, on which a large part of the present paper is based, is inspired by [20], but seems new in the present context.

Proposition 21. We have

$$
C_{0}^{T}\left(\mu_{0}, \mu_{T}\right)=\max _{v}\left(\int_{M} v_{T} d \mu_{T}-\int_{M} v_{0} d \mu_{0}\right),
$$

where the maximum is taken over the set of Lipschitz functions $v: M \times[0, T] \rightarrow \mathbb{R}$ which are $C^{1}$ on $\left.M \times\right] 0, T[$ and satisfy

$$
\left.\partial_{t} v(x, t)+H\left(x, \partial_{x} v(x, t), t\right) \leq 0 \quad \text { at each }(x, t) \in M \times\right] 0, T[.
$$

Proof. First, let $v$ be a continuous function of $M \times[0, T]$ which is differentiable on $M \times] 0, T$, where it satisfies $\left[8\right.$. Then, for each $C^{1}$ curve $\gamma:[0, T] \rightarrow M$,

$$
\begin{gathered}
\int_{0}^{T} L(\gamma(t), \dot{\gamma}(t), t) d t \geq \int_{0}^{T}\left(\partial_{x} v(\gamma(t), t) \cdot \dot{\gamma}(t)-H(\gamma(t), v(\gamma(t), t), t)\right) d t \\
\quad \geq \int_{0}^{T}\left(\partial_{x} v(\gamma(t), t) \cdot \dot{\gamma}(t)+\partial_{t} v(\gamma(t), t)\right) d t=v(\gamma(T), T)-v(\gamma(0), 0)
\end{gathered}
$$

As a consequence, $v(y, T)-v(x, 0) \leq c_{0}^{T}(x, y)$ for each $x$ and $y$, so that

$$
\int v_{T} d \mu_{T}-\int v_{0} d \mu_{0} \leq C_{0}^{T}\left(\mu_{0}, \mu_{T}\right)
$$

The converse follows directly from the next theorem, which is an analog in our context of the main result of [20].

Theorem 3. For each optimal Kantorovich pair $\left(\phi_{0}, \phi_{1}\right)$, there exists a Lipschitz function $v: M \times[0, T] \rightarrow \mathbb{R}$ which is $C^{1}$ on $\left.M \times\right] 0, T[$, coincides with $u$ on $M \times\{0, T\} \cup$ $\mathcal{T}\left(\phi_{0}, \phi_{1}\right)$, and satisfies the inequality (8) strictly at each point of $\left.M \times\right] 0, T\left[-\mathcal{T}\left(\phi_{0}, \phi_{1}\right)\right.$. 
Proof. The proof of [20] cannot be translated to our context in a straightforward way. Our proof is different, and, we believe, simpler. It is based on:

Proposition 22. There exists a function $V \in C^{2}(M \times[0, T], \mathbb{R})$ which is null on $\mathcal{T}\left(\phi_{0}, \phi_{1}\right)$, positive on $M \times] 0, T\left[-\mathcal{T}\left(\phi_{0}, \phi_{1}\right)\right.$, and such that

$$
\phi_{1}(y)=\min _{\gamma(T)=y}\left(\phi_{0}(\gamma(0))+\int_{0}^{T}(L(\gamma(t), \dot{\gamma}(t), t)-V(\gamma(t), t)) d t\right) .
$$

Proof. Define the norm

$$
\|u\|_{2}=\sum_{\theta \in \Theta}\|u \circ \theta\|_{C^{2}\left(B_{1} \times[0, T], \mathbb{R}\right)}
$$

of functions $u \in C^{2}(M \times[0, T], \mathbb{R})$, where $\Theta$ is the atlas of $M$ defined in the Appendix. Denote by $U$ the open set $M \times] 0, T\left[-\mathcal{T}\left(\phi_{0}, \phi_{1}\right)\right.$. We need a lemma.

Lemma 23. Let $U_{1} \subset U$ be an open set whose closure $\bar{U}_{1}$ is compact and contained in $U$, and let $\epsilon>0$ be given. There exists a function $V_{1} \in C^{2}(M \times[0, T], \mathbb{R})$ which is positive on $U_{1}$, null outside of $\bar{U}_{1}$, and such that 9 holds with $V=V_{1}$, and $\left\|V_{1}\right\|_{2} \leq \epsilon$.

Proof. Fix the open set $U_{1}$, the pair $\left(\phi_{0}, \phi_{1}\right)$ and $y \in M$. We claim that the minimum in

$$
\min _{\gamma(T)=y}\left(\phi_{0}(\gamma(0))+\int_{0}^{T}\left(L(\gamma(t), \dot{\gamma}(t), t)-V_{1}(\gamma(t), t)\right) d t\right)
$$

is reached at a path $\gamma$ whose graph does not meet $U_{1}$, provided that $V_{1}$ is supported in $\bar{U}_{1}$ and is sufficiently small in the $C^{0}$ topology. In order to prove the claim, suppose the contrary. There exist sequences $V_{n}^{1}(n \in \mathbb{N})$ and $\gamma_{n}$ such that

$$
\min _{\gamma(T)=y}\left(\phi_{0}(\gamma(0))+\int_{0}^{T}\left(L(\gamma(t), \dot{\gamma}(t), t)-V_{n}(\gamma(t), t)\right) d t\right)
$$

is reached at $\gamma_{n}$, the graph of $\gamma_{n}$ meets $U_{1}, V_{n}$ is supported in $\bar{U}_{1}$ (for all $n \in \mathbb{N}$ ) and $V_{n} \rightarrow 0$ in the $C^{0}$ topology. As a consequence each $\gamma_{n}$ is $C^{2}$ and the sequence $\gamma_{n}(n \in \mathbb{N})$ is a minimizing sequence for

$$
\phi_{1}(y)=\min _{\gamma(T)=y}\left(\phi_{0}(\gamma(0))+\int_{0}^{T} L(\gamma(t), \dot{\gamma}(t), t) d t\right) .
$$

Hence this sequence is compact for the $C^{2}$ topology and, by extracting a subsequence if needed, it can be assumed to converge to some $\gamma_{\infty}$. Clearly $\gamma_{\infty}$ is a minimizer for (10) with graph meeting $\bar{U}_{1}$. This contradicts $\left.\bar{U}_{1} \subset U=M \times\right] 0, T\left[-\mathcal{T}\left(\phi_{0}, \phi_{1}\right)\right.$ and the fact that the graph of $\gamma_{\infty}$ is included in $\overline{\mathcal{T}\left(\phi_{0}, \phi_{1}\right)}$ (see Definition 18 ).

Let $U_{n} \subset U, n \in \mathbb{N}$, be a sequence of open sets covering $U$ with closures contained in $U$. There exists a sequence of functions $V_{n} \in C^{2}(M \times[0, T], \mathbb{R})$ such that, for each $n \in \mathbb{N}$ : 
- $V_{n}$ is positive in $U_{n}$ and null outside of $\bar{U}_{n}$.

- $\left\|V_{n}\right\|_{2} \leq 2^{-n} \epsilon$.

- The equality 99 holds for the function $V^{n}=\sum_{i=1}^{n} V_{i}$.

Such a sequence can be build inductively by applying Lemma 23 to the Lagrangian $L-$ $V^{n-1}$ with $\epsilon_{n}=2^{-n} \epsilon$. Since $\left\|V_{n}\right\| \leq 2^{-n} \epsilon$, the sequence $V^{n}$ converges in $C^{2}$ norm to a limit $V \in C^{2}(M \times[0, T], \mathbb{R})$. This function $V$ has the desired properties. The proposition is proved.

In order to finish the proof of the theorem, we shall consider the new Lagrangian $\tilde{L}=$ $L-V$, and the associated Hamiltonian $\tilde{H}=H+V$, as well as the associated cost functions $\tilde{c}_{s}^{t}$. Let

$$
\tilde{u}(x, t):=\min _{y \in M}\left(\phi_{0}(y)+\tilde{c}_{0}^{t}(y, x)\right)
$$

be the viscosity solution of the Hamilton-Jacobi equation

$$
\partial_{t} \tilde{u}+H\left(x, \partial_{x} \tilde{u}, t\right)=-V(x, t)
$$

emanating from $\phi_{0}$. The equality $(9)$ says that $\tilde{u}_{T}=\phi_{1}=u_{T}$. The function $\tilde{u}$ is Lipschitz on $M \times[0, T]$, as a viscosity solution of $(\tilde{H} J)$ emanating from a Lipschitz function. It is obviously a viscosity subsolution of $(H J)$, which is strict outside of $M \times\{0, T\} \cup$ $\mathcal{T}\left(\phi_{0}, \phi_{1}\right)$ (where $V$ is positive). This means that the inequality $(8)$ is strict at each point of differentiability of $\tilde{u}$ outside of $M \times\{0, T\} \cup \mathcal{T}\left(\phi_{0}, \phi_{1}\right)$. We have $\breve{u} \leq \tilde{u} \leq u$, this relation being satisfied by each viscosity subsolution of $(H J)$ which satisfies $u_{0}=\phi_{0}$ and $u_{T}=\phi_{1}$. As a consequence, we have $\breve{u}=\tilde{u}=u$ on $\mathcal{T}\left(\phi_{0}, \phi_{1}\right)$, and $\tilde{u}$ is differentiable at each point of $\mathcal{T}\left(\phi_{0}, \phi_{1}\right)$. Furthermore, $d u=d \tilde{u}=d \breve{u}$ on this set. We then obtain the desired function $v$ of the theorem from $\tilde{u}$ by regularization, applying Theorem 9.2 of [20].

\section{Optimal objects of the direct problems}

We now prove Theorem A as well as the results of Section 2 The following lemma generalizes a result of Benamou and Brenier [6].

Lemma 24. We have

$$
C_{0}^{T}\left(\mu_{0}, \mu_{T}\right)=\min _{m_{0} \in \mathcal{I}\left(\mu_{0}, \mu_{T}\right)} A\left(m_{0}\right)=\min _{m \in \mathcal{M}\left(\mu_{0}, \mu_{T}\right)} A(m)=\min _{\chi \in \mathcal{C}\left(\mu_{0}, \mu_{T}\right)} A(\chi) .
$$

Moreover $\chi(d v)=A(\chi)$ for every optimal $\chi$, where $v$ is given by Theorem 3

Proof. In view of Lemma 11, it is enough to prove that, for each transport current $\chi \in$ $\mathcal{C}\left(\mu_{0}, \mu_{T}\right)$, we have $A(\chi) \geq C_{0}^{T}\left(\mu_{0}, \mu_{T}\right)$. Let $v: M \times[0, T] \rightarrow \mathbb{R}$ be a Lipschitz subsolution of $(H J)$ which is $C^{1}$ on $\left.M \times\right] 0, T$, and such that $\left(v_{0}, v_{T}\right)$ is an optimal Kantorovich pair. For each current $\chi \in \mathcal{C}\left(\mu_{0}, \mu_{T}\right)$, we have $A(\chi) \geq \chi(d v)=C_{0}^{T}\left(\mu_{0}, \mu_{T}\right)$, which ends the proof.

From now on we fix: 
- An optimal Kantorovich pair $\left(\phi_{0}, \phi_{1}\right)$.

- A Lipschitz subsolution $v: M \times[0, T] \rightarrow \mathbb{R}$ of the Hamilton-Jacobi equation which satisfies $v_{0}=\phi_{0}$ and $v_{T}=\phi_{1}$ and which is $C^{1}$ on $\left.M \times\right] 0, T[$.

- A bounded vector field $X: M \times] 0, T[\rightarrow T M$ which is locally Lipschitz and satisfies

$$
X(x, t)=\partial_{p} H\left(x, \partial_{x} v(x, t), t\right) \quad \text { on } \mathcal{T}\left(\phi_{0}, \phi_{1}\right) .
$$

\subsection{Characterization of optimal currents}

Each optimal transport current $\chi$ can be written as

$$
\chi=(X, 1) \wedge \mu_{\chi},
$$

with a measure $\mu_{\chi}$ concentrated on $\mathcal{T}\left(\phi_{0}, \phi_{1}\right)$. The current $\chi$ is then Lipschitz regular, so that there exists a transport interpolation $\mu_{t}, t \in[0, T]$, such that $\mu_{\chi}=\mu_{t} \otimes d t$ (see Appendix) and $\mu_{t}=\left(\Psi_{s}^{t}\right)_{\sharp} \mu_{s}$ for each $s$ and $t$ in $] 0, T[$.

Proof. Let $\chi$ be an optimal transport current, that is, a transport current $\chi \in \mathcal{C}\left(\mu_{0}, \mu_{T}\right)$ such that $A(\chi)=C_{0}^{T}\left(\mu_{0}, \mu_{T}\right)$. Recall the definition of the action $A(\chi)$ that will be used here:

$$
A(\chi)=\sup _{\omega \in \Omega^{0}}\left(\chi\left(\omega^{x}, 0\right)-\int_{M \times[0, T]} H\left(x, \omega^{x}(x, t), t\right) d \mu_{\chi}\right) .
$$

Since $H\left(x, \partial_{x} v, t\right)+\partial_{t} v \leq 0$, we have

$$
\begin{aligned}
A(\chi) & =\chi(d v) \leq \chi(d v)-\int\left(H\left(x, \partial_{x} v(x, t), t\right)+\partial_{t} v\right) d \mu_{\chi} \\
& =\chi\left(\partial_{x} v, 0\right)-\int H\left(x, \partial_{x} v(x, t), t\right) d \mu_{\chi} .
\end{aligned}
$$

The other inequality holds by the definition of $A$, so that

$$
\begin{aligned}
\chi(d v) & =\chi(d v)-\int\left(H\left(x, \partial_{x} v(x, t), t\right)+\partial_{t} v\right) d \mu_{\chi} \\
& =\chi\left(\partial_{x} v, 0\right)-\int H\left(x, \partial_{x} v(x, t), t\right) d \mu_{\chi},
\end{aligned}
$$

and we conclude that $H\left(x, \partial_{x} v(x, t), t\right)+\partial_{t} v$ vanishes on the support of $\mu_{\chi}$, or in other words the measure $\mu_{\chi}$ is concentrated on $\mathcal{T}\left(\phi_{0}, \phi_{1}\right)$. In addition, for all forms $\omega=\omega^{x}+$ $\omega^{t} d t$, we have

$\chi\left(\partial_{x} v+\omega^{x}, 0\right)-\int H\left(x, \partial_{x} v+\omega^{x}, t\right) d \mu_{\chi} \leq \chi\left(\partial_{x} v, 0\right)-\int H\left(x, \partial_{x} v, t\right) d \mu_{\chi}=A(\chi)$.

Hence

$$
\chi\left(\omega^{x}, 0\right)=\int \partial_{p} H\left(x, \partial_{x} v, t\right)\left(\omega^{x}\right) d \mu_{\chi}
$$


for each form $\omega$. This equality can be rewritten as

$$
\chi(\omega)=\int\left(\partial_{p} H\left(x, \partial_{x} v, t\right)\left(\omega^{x}\right)+\omega^{t}\right) d \mu_{\chi}
$$

which precisely says that

$$
\chi=\left(\partial_{p} H\left(x, \partial_{x} v(x, t), t\right), 1\right) \wedge \mu_{\chi}=(X, 1) \wedge \mu_{\chi} .
$$

The last equality follows from the fact that the vector fields $X$ and $\partial_{p} H\left(x, \partial_{x} v(x, t), t\right)$ are equal on the support of $\mu_{\chi}$. By the structure of Lipschitz regular transport currents, we obtain the existence of a continuous family $\mu_{t}, t \in[0, T]$, of probability measures such that $\mu_{\chi}=\mu_{t} \otimes d t$ and $\mu_{t}=\left(\Psi_{s}^{t}\right)_{\sharp} \mu_{s}$ for each $s$ and $t$ in ]0, $T$ [. Since the restriction to a subinterval $[s, t] \subset[0, T]$ of an optimal transport current $\chi$ is clearly an optimal transport current for the transportation problem between $\mu_{s}$ and $\mu_{t}$ with $\operatorname{cost} c_{s}^{t}$, we conclude that the path $\mu_{t}$ is a transport interpolation.

\subsection{Characterization of transport interpolations}

Each transport interpolation $\mu_{t}$ satisfies

$$
\mu_{t}=\left(\Psi_{s}^{t}\right)_{\sharp} \mu_{s}
$$

for each $(s, t) \in] 0, T\left[^{2}\right.$. The mapping

$$
\mu_{t} \mapsto(X, 1) \wedge\left(\mu_{t} \otimes d t\right)
$$

is a bijection between the set of transport interpolations and the set of optimal transport currents.

Proof. We fix a transport interpolation $\mu_{t}$ and two times $s<s^{\prime}$ in $] 0, T$ [. Let $\chi_{1}$ be a transport current on $M \times[0, s]$ between the measures $\mu_{0}$ and $\mu_{s}$ which is optimal for the $\operatorname{cost} c_{0}^{s}$, let $\chi_{2}$ be a transport current on $M \times\left[s, s^{\prime}\right]$ between $\mu_{s}$ and $\mu_{s^{\prime}}$ which is optimal for $c_{s}^{s^{\prime}}$, and let $\chi_{3}$ be a transport current on $M \times\left[s^{\prime}, T\right]$ between $\mu_{s^{\prime}}$ and $\mu_{T}$ which is optimal for $c_{s^{\prime}}^{T}$. Then the current $\chi$ on $M \times[0, T]$ which coincides with $\chi_{1}$ on $M \times[0, s]$, with $\chi_{2}$ on $M \times\left[s, s^{\prime}\right]$ and with $\chi_{3}$ on $\left[s^{\prime}, T\right]$ belongs to $\mathcal{C}\left(\mu_{0}, \mu_{T}\right)$. In addition, since $\mu_{t}$ is a transport interpolation, we have

$$
A(\chi)=C_{0}^{s}\left(\mu_{0}, \mu_{s}\right)+C_{s}^{s^{\prime}}\left(\mu_{s}, \mu_{s^{\prime}}\right)+C_{s^{\prime}}^{T}\left(\mu_{s^{\prime}}, \mu_{T}\right)=C_{0}^{T}\left(\mu_{0}, \mu_{T}\right)
$$

Hence $\chi$ is an optimal transport current for the $\operatorname{cost} c_{0}^{T}$. In view of the characterization of optimal currents, this implies that $\chi=(X, 1) \wedge \mu_{\chi}$, and

$$
\mu_{\chi}=\left(\left(\Psi_{s}^{t}\right)_{\sharp} \mu_{s}\right) \otimes d t=\left(\left(\Psi_{s^{\prime}}^{t}\right)_{\sharp} \mu_{s^{\prime}}\right) \otimes d t .
$$


By uniqueness of the continuous desintegration of $\mu_{\chi}$, we deduce that, for each $\left.t \in\right] 0, T$, $\left(\Psi_{s}^{t}\right)_{\sharp} \mu_{s}=\left(\Psi_{s^{\prime}}^{t}\right)_{\sharp} \mu_{s^{\prime}}$, and since this holds for all $s$ and $s^{\prime}$, we have $\left(\Psi_{s}^{t}\right)_{\sharp} \mu_{s}=\mu_{t}$ for all $(s, t) \in] 0, T\left[^{2}\right.$. It follows that $\chi=(X, 1) \wedge\left(\mu_{t} \otimes d t\right)$. We have proved that the mapping

$$
\mu_{t} \mapsto(X, 1) \wedge\left(\mu_{t} \otimes d t\right)
$$

associates an optimal transport current to each transport interpolation. This mapping is obviously injective, and it is surjective in view of the characterization of optimal currents.

\subsection{Characterization of optimal measures}

The mapping

$$
\chi \mapsto(X \times \tau)_{\sharp} \mu_{\chi}
$$

is a bijection between the set of optimal transport currents and the set of optimal transport measures ( $\tau: M \times[0, T] \rightarrow[0, T]$ is the projection on the second factor; see Appendix). Each optimal transport measure is thus invariant (see (4) and Definition 5). The mapping

$$
m_{0} \mapsto \mu_{t}=\left(\pi \circ \psi_{0}^{t}\right)_{\sharp} m_{0}
$$

is a bijection between the set of optimal initial measures $m_{0}$ and the set of interpolations. An invariant measure $m$ is optimal if and only if it is supported on the set $\tilde{\mathcal{T}}\left(\phi_{0}, \phi_{1}\right)$.

Proof. If $m$ is an optimal transport measure, then the associated current $\chi_{m}$ is an optimal transport current, and $A(m)=A\left(\chi_{m}\right)$. Let $\mu_{m}$ be the time component of $\chi_{m}$, which is also the measure $(\pi \times \tau)_{\sharp} m$. In view of the characterization of optimal currents, we have $\chi_{m}=(X, 1) \wedge \mu_{m}$. We claim that the equality $A\left(\chi_{m}\right)=A(m)$ implies that $m$ is supported on the graph of $X$. Indeed, we have the pointwise inequality

$$
\partial_{x} v(x, t) \cdot V-H\left(x, \partial_{x} v(x, t), t\right) \leq L(x, V, t)
$$

for each $(x, V, t) \in T M \times] 0, T[$. Integrating with respect to $m$, we get

$$
\begin{aligned}
A\left(\chi_{m}\right)=\chi_{m}(d v) & =\int_{T M \times[0, T]}\left(\partial_{x} v(x, t) \cdot V+\partial_{t} v(x, t)\right) d m(x, V, t) \\
& =\int_{T M \times[0, T]}\left(\partial_{x} v(x, t) \cdot V-H\left(x, \partial_{x} v(x, t), t\right)\right) d m(x, V, t) \\
& =\int_{M \times[0, T]} L(x, V, t) d m(x, V, t)=A(m),
\end{aligned}
$$

which means that $m$ is concentrated on the set where the inequality (12) is an equality, that is, on the graph of the vector field $\partial_{p} H\left(x, \partial_{x} v(x, t), t\right)$. Since $\mu_{m}$ is supported on $\mathcal{T}$, the measure $m$ is supported on $\tilde{\mathcal{T}}$ and satisfies $m=(X \times \tau)_{\sharp} \mu_{m}$. Let $\mu_{t}$ be the transport 
interpolation such that $\mu_{m}=\mu_{t} \otimes d t$. Setting $m_{t}=\left(X_{t}\right)_{\sharp} \mu_{t}$, we have $m=m_{t} \otimes d t$. Observing that

$$
X_{t} \circ \Psi_{s}^{t}=\psi_{s}^{t} \circ X_{s}
$$

on $\mathcal{T}_{s}$, we conclude, since $\mu_{s}$ is supported on $\mathcal{T}_{s}$, that

$$
\left(\psi_{s}^{t}\right)_{\sharp} m_{s}=m_{t},
$$

which means that the measure $m$ is invariant.

Conversely, let $m=m_{t} \otimes d t$ be an invariant measure supported on $\tilde{\mathcal{T}}\left(\phi_{0}, \phi_{1}\right)$. We have

$$
A(m)=\int_{0}^{T} \int_{T M} L(x, v, t) d m_{t}(x, v) d t=\int_{0}^{T} \int_{T M} L\left(\psi_{0}^{t}(x, v), t\right) d m_{0}(x, v) d t,
$$

and by Fubini,

$$
\begin{aligned}
A(m) & =\int_{T M} \int_{0}^{T} L\left(\psi_{0}^{t}(x, v), t\right) d t d m_{0}(x, v) \\
& =\int_{T M}\left(\phi_{1}\left(\pi \circ \psi_{0}^{T}(x, v)\right)-\phi_{0}(x)\right) d m_{0}(x, v),
\end{aligned}
$$

and since $m_{0}$ is an initial transport measure, we get

$$
A(m)=\int_{T M} \phi_{1} d \mu_{T}-\int_{T M} \phi_{0} d \mu_{0}=C_{0}^{T}\left(\mu_{0}, \mu_{T}\right) .
$$

\section{Absolute continuity}

In this section, we make the additional assumption that the initial measure $\mu_{0}$ is absolutely continuous, and prove Theorem B. The following lemma answers a question asked to us by Cedric Villani.

Lemma 25. If $\mu_{0}$ or $\mu_{T}$ is absolutely continuous with respect to the Lebesgue class, then each interpolating measure $\left.\mu_{t}, t \in\right] 0, T[$, is absolutely continuous.

Proof. If $\mu_{t}, t \in[0, T]$, is a transport interpolation, we have proved that

$$
\mu_{t}=\left(\pi \circ \psi_{s}^{t} \circ X_{S}\right)_{\sharp} \mu_{s}
$$

for all $s \in] 0, T\left[\right.$ and $t \in[0, T]$. Since the function $\pi \circ \psi_{t}^{s} \circ X_{t}$ is Lipschitz, it maps Lebesgue zero measure sets into Lebesgue zero measure sets, and so it transports singular measures into singular measures. It follows that if, for some $s \in] 0, T\left[\right.$, the measure $\mu_{s}$ is not absolutely continuous, then none of the measures $\mu_{t}, t \in[0, T]$, are absolutely continuous.

In order to continue the investigation of the specific properties satisfied when $\mu_{0}$ is absolutely continuous, we first need some more general results. Let $\left(\phi_{0}, \phi_{1}\right)$ be an optimal 
Kantorovich pair for the measures $\mu_{0}$ and $\mu_{T}$ and for the cost $c_{0}^{T}$. Recall that we have defined $\mathcal{F}\left(\phi_{0}, \phi_{1}\right) \subset C^{2}([0, T], M)$ as the set of curves $\gamma$ such that

$$
\phi_{1}(\gamma(T))=\phi_{0}(\gamma(0))+\int_{0}^{T} L(\gamma(t), \dot{\gamma}(t), t) d t .
$$

Let $\mathcal{F}_{0}\left(\phi_{0}, \phi_{1}\right)$ be the set of initial velocities $(x, v) \in T M$ such that the curve $t \mapsto \pi \circ$ $\psi_{0}^{t}(x, v)$ belongs to $\mathcal{F}\left(\phi_{0}, \phi_{1}\right)$. Note that there is a natural bijection between $\mathcal{F}_{0}\left(\phi_{0}, \phi_{1}\right)$ and $\mathcal{F}\left(\phi_{0}, \phi_{1}\right)$.

Lemma 26. The set $\mathcal{F}_{0}\left(\phi_{0}, \phi_{1}\right)$ is compact. The maps $\pi$ and $\pi \circ \psi_{0}^{T}: \mathcal{F}_{0}\left(\phi_{0}, \phi_{1}\right) \rightarrow M$ are surjective. If $x$ is a point of differentiability of $\phi_{0}$, then the set $\pi^{-1}(x) \cap \mathcal{F}_{0}\left(\phi_{0}, \phi_{1}\right)$ contains a single point. There exists a Borel measurable set $\Sigma \subset M$ of full measure, whose points are points of differentiability of $\phi_{0}$, and such that the map

$$
x \mapsto S(x)=\pi^{-1}(x) \cap \mathcal{F}_{0}\left(\phi_{0}, \phi_{1}\right)
$$

is Borel measurable on $\Sigma$.

Proof. The compactness of $\mathcal{F}_{0}\left(\phi_{0}, \phi_{1}\right)$ follows from the fact, already mentioned, that the set of minimizing extremals $\gamma:[0, T] \rightarrow M$ is compact for the $C^{2}$ topology.

It is equivalent to say that the projection $\pi$ restricted to $\mathcal{F}_{0}\left(\phi_{0}, \phi_{1}\right)$ is surjective, and, for each $x \in M$, there exists a curve emanating from $x$ in $\mathcal{F}\left(\phi_{0}, \phi_{1}\right)$. In order to build such curves, recall that

$$
\phi_{0}(x)=\max _{\gamma}\left(\phi_{1}(\gamma(T))-\int_{0}^{T} L(\gamma(t), \dot{\gamma}(t), t) d t\right)
$$

where the maximum is taken over the set of curves which satisfy $\gamma(0)=x$. Any maximizing curve is then a curve in $\mathcal{F}\left(\phi_{0}, \phi_{1}\right)$ which satisfies $\gamma(0)=x$. In order to prove that the map $\pi \circ \psi_{0}^{T}$ restricted to $\mathcal{F}_{0}\left(\phi_{0}, \phi_{1}\right)$ is surjective, it is sufficient to build, for each $x \in M$, a curve in $\mathcal{F}\left(\phi_{0}, \phi_{1}\right)$ which ends at $x$. Such a curve is obtained as a minimizer in the expression

$$
\phi_{1}(x)=\min _{\gamma}\left(\phi_{0}(\gamma(0))+\int_{0}^{T} L(\gamma(t), \dot{\gamma}(t), t) d t\right) .
$$

Now consider a point $x$ of differentiability of $\phi_{0}$. Applying the general result on the differentiability of viscosity solutions to the backward viscosity solution $\breve{u}$, we find that there exists a unique maximizer to the problem

$$
\phi_{0}(x)=\max _{\gamma}\left(\phi_{1}(\gamma(T))-\int_{0}^{T} L(\gamma(t), \dot{\gamma}(t), t) d t\right)
$$

and that this maximizer is the extremal with initial condition $\left(x, \partial_{p} H\left(x, d \phi_{0}(x), 0\right)\right)$. As a consequence, there exists a single point $S(x)$ in $\mathcal{F}_{0}\left(\phi_{0}, \phi_{1}\right)$ above $x$, and in addition we have the explicit expression

$$
S(x)=\partial_{p} H\left(x, d \phi_{0}(x), 0\right) .
$$


Since the set of points of differentiability of $\phi_{0}$ has total Lebesgue measure-because $\phi_{0}$ is Lipschitz-there exists a sequence $K_{n}$ of compact sets such that $\phi_{0}$ is differentiable at each point of $K_{n}$ and the Lebesgue measure of $M-K_{n}$ converges to zero. For each $n$, the set $\pi^{-1}\left(K_{n}\right) \cap \mathcal{F}_{0}\left(\phi_{0}, \phi_{1}\right)$ is compact, and the restriction to this set of the canonical projection $\pi$ is injective and continuous. It follows that the inverse function $S$ is continuous on $K_{n}$. As a consequence, $S$ is Borel measurable on $\Sigma:=\bigcup_{n} K_{n}$.

Lemma 27. The initial transport measure $m_{0}$ is optimal if and only if it is an initial transport measure supported on $\mathcal{F}_{0}\left(\phi_{0}, \phi_{1}\right)$.

Proof. This statement is a reformulation of the result in 4.3 stating that the optimal transport measures are the invariant measures supported on $\mathcal{T}\left(\phi_{0}, \phi_{1}\right)$.

Proposition 28. If $\mu_{0}$ is absolutely continuous, then there exists a unique optimal initial measure $m_{0}$. There exists a Borel section $S: M \rightarrow T M$ of the canonical projection such that $m_{0}=S_{\sharp} \mu_{0}$, and this section is unique $\mu_{0}$-almost everywhere. For each $t \in[0, T]$, the map $\pi \circ \psi_{0}^{t} \circ S: M \rightarrow M$ is then an optimal transport map between $\mu_{0}$ and $\mu_{t}$.

Proof. Let $S: \Sigma \rightarrow T M$ be the Borel map constructed in Lemma 26. For convenience, we shall also denote by $S$ the same map extended by zero outside of $\Sigma$, which is a Borel section $S: M \rightarrow T M$. Since the set $\Sigma$ is of full Lebesgue measure, and since the measure $\mu_{0}$ is absolutely continuous, we have $\mu_{0}(\Sigma)=1$. Consider the measure $m_{0}=S_{\sharp}\left(\mu_{0 \mid \Sigma}\right)$. This is a probability measure on $T M$ which is concentrated on $\mathcal{F}_{0}\left(\phi_{0}, \phi_{1}\right)$ and satisfies $\pi_{\sharp} m_{0}=\mu_{0}$. We claim that it is the only measure with these properties. Indeed, if $\tilde{m}_{0}$ is a measure with these properties, then $\pi_{\sharp} \tilde{m}_{0}=\mu_{0}$, hence $\tilde{m}_{0}$ is concentrated on $\pi^{-1}(\Sigma) \cap$ $\mathcal{F}_{0}\left(\phi_{0}, \phi_{1}\right)$. But then, since $\pi$ induces a Borel isomorphism from $\pi^{-1}(\Sigma) \cap \mathcal{F}_{0}\left(\phi_{0}, \phi_{1}\right)$ onto its image $\Sigma$, with inverse $S$, we must have $\tilde{m}_{0}=S_{\sharp} \mu_{0}$. As a consequence, $m_{0}=$ $S_{\sharp} \mu_{0}$ is the only candidate to be an optimal initial transport measure. Since we have already proved the existence of an optimal initial transport measure, this implies that $m_{0}$ is the only optimal initial transport measure. Of course, we could prove directly that $m_{0}$ is an initial transport measure, but as we have seen, this is not necessary.

\subsection{Remark}

That there exists an optimal transport map if $\mu_{0}$ is absolutely continuous could be proved directly as a consequence of the following properties of the cost function.

Lemma 29. The cost function $c_{0}^{T}(x, y)$ is semiconcave on $M \times M$. In addition, we have the following injectivity property for each $x \in M$ : If the differentials $\partial_{x} c_{0}^{T}(x, y)$ and $\partial_{x} c_{0}^{T}\left(x, y^{\prime}\right)$ exist and are equal, then $y=y^{\prime}$.

In view of these properties of the cost function, it is not hard to prove the following lemma using an optimal Kantorovich pair in the spirit of works of Brenier [11] and Carlier [15].

Lemma 30. There exists a compact subset $K \subset M \times M$ such that the fiber $K_{x}=K \cap$ $\pi_{0}^{-1}(x)$ is a single point for Lebesgue almost every $x$, and such that $K$ contains the support of all optimal plans. 
The proof of the existence of an optimal map for an absolutely continuous measure $\mu_{0}$ can then be terminated using the following result (see [1, Proposition 2.1]).

Proposition 31. A transport plan $\eta$ is induced from a transport map if and only if it is concentrated on an $\eta$-measurable graph.

\subsection{Remark}

Assuming only that $\mu_{0}$ vanishes on countably $(d-1)$-rectifiable sets, we can conclude that the same property holds for all interpolating measures $\mu_{t}, t<T$, and that the assertion of Proposition 28 holds. This is proved almost identically. The only refinement needed is that the set of singular points of the semiconvex function $\phi_{0}$ is countably $(d-1)$-rectifiable (see [13]).

\section{Aubry-Mather theory}

We explain the relations between the results obtained so far and Mather theory, and prove Theorem C. Up to now, we have worked with fixed measures $\mu_{0}$ and $\mu_{T}$. Let us study the optimal value $C_{0}^{T}\left(\mu_{0}, \mu_{T}\right)$ as a function of the measures $\mu_{0}$ and $\mu_{T}$.

Lemma 32. The function

$$
\left(\mu_{0}, \mu_{T}\right) \mapsto C_{0}^{T}\left(\mu_{0}, \mu_{T}\right)
$$

is convex and lower semicontinuous on the set of pairs of probability measures on $M$.

Proof. This follows directly from the expression

$$
C_{0}^{T}\left(\mu_{0}, \mu_{T}\right)=\max _{\left(\phi_{0}, \phi_{1}\right)}\left(\int_{M} \phi_{1} d \mu_{T}-\int_{M} \phi_{0} d \mu_{0}\right)
$$

as a maximum of continuous linear functions.

From now on, we assume that the Lagrangian $L$ is defined for all times, $L \in C^{2}(T M \times$ $\mathbb{R}, \mathbb{R})$, and satisfies

$$
L(x, v, t+1)=L(x, v, t)
$$

in addition to the standing hypotheses. Let us restate Theorem $\mathrm{C}$ with more details. Recall that $\alpha$ is the action of Mather measures, as defined in the introduction.

Theorem $\mathbf{C}^{\prime}$. There exists a Lipschitz vector field $X_{0}$ on $M$ such that all the Mather measures are supported on the graph of $X_{0}$. We have

$$
\alpha=\min _{\mu} C_{0}^{1}(\mu, \mu)
$$

where the minimum is taken over the set of probability measures on $M$. The mapping $m_{0} \mapsto \pi_{\sharp} m_{0}$ is a bijection between the set of Mather measures $m_{0}$ and the set of probability measures $\mu$ on $M$ satisfying $C_{0}^{1}(\mu, \mu)=\alpha$. More precisely, if $\mu$ is such a probability measure, then there exists a unique initial transport measure $m_{0}$ for the transport 
problem between $\mu_{0}=\mu$ and $\mu_{1}=\mu$ with cost $c_{0}^{1}$; this measure is $m_{0}=\left(X_{0}\right)_{\sharp} \mu$, and it is a Mather measure.

The proof, and related digressions, occupy the rest of the section.

Lemma 33. The minima

$$
\alpha_{T}:=\min _{\mu \in \mathcal{B}_{1}(M)} \frac{1}{T} C_{0}^{T}(\mu, \mu), \quad T \in \mathbb{N},
$$

exist and are all equal. In addition, any measure $\mu^{1} \in \mathcal{B}_{1}(M)$ which minimizes $C_{0}^{1}(\mu, \mu)$ also minimizes $C_{0}^{T}(\mu, \mu)$ for all $T \in \mathbb{N}$.

Proof. The existence of the minima follows from the compactness of the set of probability measures and from the semicontinuity of the function $C_{0}^{T}$. Let $\mu^{1}$ be a minimizing measure for $\alpha_{1}$ and let $m^{1}$ be an optimal transport measure for the transportation problem $C_{0}^{1}\left(\mu^{1}, \mu^{1}\right)$. Let $m^{T}$ be the measure on $T M \times[0, T]$ obtained by concatenating $T$ translated versions of $m^{1}$. This means that $m^{T}$ is the only measure on $T M \times[0, T]$ whose restriction to $T M \times[i, i+1]$ is obtained by translation from $m$, for each integer $i$. It is easy to check that $m^{T}$ is indeed a transport measure between $\mu_{0}=\mu^{1}$ and $\mu_{T}=\mu^{1}$ on the time interval $[0, T]$, and that $A_{0}^{T}\left(m^{T}\right)=T A_{0}^{1}\left(m^{1}\right)$. As a consequence, we have

$$
T \alpha_{T} \leq C_{0}^{T}\left(\mu^{1}, \mu^{1}\right) \leq A_{0}^{T}\left(m^{T}\right)=T C_{0}^{1}\left(\mu^{1}, \mu^{1}\right)=T \alpha_{1},
$$

which implies $\alpha_{T} \leq \alpha_{1}$.

Let us now prove that $\alpha_{T} \geq \alpha_{1}$. In order to do so, we consider an optimal measure $\mu^{T}$ for $\alpha_{T}$, and consider a transport interpolation $\mu_{t}^{T}, t \in[0, T]$, between the measures $\mu_{0}=\mu^{T}$ and $\mu_{T}=\mu^{T}$. Consider, for $t \in[0,1]$, the measure

$$
\tilde{\mu}_{t}^{T}:=\frac{1}{T} \sum_{i=0}^{T-1} \mu_{t+i}^{T},
$$

and note that $T \tilde{\mu}_{0}^{T}=\mu_{0}^{T}+\sum_{i=1}^{T-1} \mu_{i}^{T}=\mu_{T}^{T}+\sum_{i=1}^{T-1} \mu_{i}^{T}=T \tilde{\mu}_{1}^{T}$. In view of the convexity of $C_{0}^{1}$,

$$
\begin{aligned}
C_{0}^{1}\left(\tilde{\mu}_{0}^{T}, \tilde{\mu}_{1}^{T}\right) & =C_{0}^{1}\left(\frac{1}{T} \sum_{i=0}^{T-1}\left(\mu_{i}^{T}, \mu_{i+1}^{T}\right)\right) \leq \frac{1}{T} \sum_{i=0}^{T-1} C_{i}^{i+1}\left(\mu_{i}^{T}, \mu_{i+1}^{T}\right) \\
& =\frac{1}{T} C_{0}^{T}\left(\mu^{T}, \mu^{T}\right)=\alpha_{T} .
\end{aligned}
$$

Since $\tilde{\mu}_{0}^{T}=\tilde{\mu}_{1}^{T}$, this implies that $\alpha_{1} \leq \alpha_{T}$, as desired.

Lemma 34. We have $\alpha_{1} \leq \alpha$.

Proof. If $m_{0}$ is a Mather measure, then it is an initial measure for the transport problem between $\mu_{0}=\pi_{\sharp} m_{0}$ and $\mu_{1}=\pi_{\sharp} m_{0}$ for the cost $c_{0}^{1}$. As a consequence, we have $\alpha=$ $A_{0}^{1}\left(m_{0}\right) \geq C_{0}^{1}\left(\mu_{0}, \mu_{0}\right) \geq \alpha_{1}$. 
Lemma 35. Let $\mu^{1}$ be a probability measure on $M$ such that $C_{0}^{1}\left(\mu^{1}, \mu^{1}\right)=\alpha_{1}$. Then there exists a unique initial transport measure $m_{0}$ for the transportation problem between $\mu_{0}=\mu^{1}$ and $\mu_{1}=\mu^{1}$ for the cost $c_{0}^{1}$. This measure satisfies $\left(\psi_{0}^{1}\right)_{\sharp} m_{0}=m_{0}$. We have $\alpha_{1}=A_{0}^{1}\left(m_{0}\right) \geq \alpha$, so that $\alpha=\alpha_{1}$ and $m_{0}$ is a Mather measure. There exists a constant $K$, which depends only on $L$, such that $m_{0}$ is supported on the graph of a K-Lipschitz vector field.

Proof. Fix a probability measure $\mu^{1}$ on $M$ such that $C_{0}^{1}\left(\mu^{1}, \mu^{1}\right)=\alpha_{1}$. Let $X: M \times$ $[0,2] \rightarrow T M$ be a vector field associated to the transport problem $C_{0}^{2}\left(\mu^{1}, \mu^{1}\right)$ by Theorem A. Note that $X_{1}$ is Lipschitz on $M$ with a Lipschitz constant $K$ which does not depend on $\mu_{1}$. We choose $X$ once and for all and fix it.

To each optimal transport measure $m^{1}$ for the transport problem $C_{0}^{1}\left(\mu^{1}, \mu^{1}\right)$, we associate the transport measure $m^{2}$ on $T M \times[0,2]$ obtained by concatenation of two translated versions of $m^{1}$, as in the proof of Lemma 33 . We have

$$
A_{0}^{2}\left(m^{2}\right)=2 A_{0}^{1}\left(m^{1}\right)=2 \alpha_{1}=2 \alpha_{2}=C_{0}^{2}\left(\mu^{1}, \mu^{1}\right) .
$$

The measure $m^{2}$ is thus an optimal transport measure for the transportation problem $C_{0}^{2}\left(\mu^{1}, \mu^{1}\right)$. Let $m_{t}, t \in[0,2]$, be the continuous family of probability measures on $T M$ such that $m^{2}=m_{t} \otimes d t$. Note that $m_{t}=\left(\psi_{s}^{t}\right)_{\sharp} m_{s}$ for all $s$ and $t$ in [0, 2], and that $m_{0}$ is the initial transport measure for the transportation problem $C_{0}^{1}\left(\mu^{1}, \mu^{1}\right)$ associated to $m^{1}$. Since $m^{2}$ was obtained by concatenation of two translated versions of the same measure $m^{1}$, we must have $m_{t+1}=m_{t}$ for almost all $\left.t \in\right] 0,1$ [, and, by continuity, $m_{0}=m_{1}=m_{2}$. This implies that $m_{0}=\left(\psi_{0}^{1}\right)_{\sharp} m_{0}$. Finally, the characterization of optimal measures implies that $m_{0}=m_{1}=\left(X_{1}\right)_{\sharp} \mu^{1}$. We have proved that $\left(X_{1}\right)_{\sharp} \mu^{1}$ is the only optimal initial transport measure for the transportation problem $C_{0}^{1}\left(\mu^{1}, \mu^{1}\right)$.

Proof of Theorem C. Let $m_{0}$ be a Mather measure, and let $\mu_{0}=\pi_{\sharp} m_{0}$. Note that we also have $\mu_{0}=\left(\pi \circ \psi_{0}^{1}\right)_{\sharp} m_{0}$. As a consequence, $m_{0}$ is an initial transport measure for the transport between $\mu_{0}$ and $\mu_{0}$ for the cost $c_{0}^{1}$, and we have

$$
\alpha=A_{0}^{1}\left(m_{0}\right) \geq C_{0}^{1}\left(\mu_{0}, \mu_{0}\right) \geq \alpha_{1} .
$$

Since $\alpha_{1}=\alpha$, all these inequalities are equalities, so that $m_{0}$ is an optimal initial transport measure, and $C_{0}^{1}\left(\mu_{0}, \mu_{0}\right)=\alpha_{1}$. It follows from Lemma 35 that $m_{0}$ is supported on the graph of a $K$-Lipschitz vector field.

Up to now, we have proved that each Mather measure is supported on the graph of a $K$-Lipschitz vector field. It remains to prove that all Mather measures are supported on a single $K$-Lipschitz graph. In order to do this, denote by $\tilde{\mathcal{M}} \subset T M$ the union of the supports of Mather measures. If $(x, v)$ and $\left(x^{\prime}, v^{\prime}\right)$ are two points of $\tilde{\mathcal{M}}$, then there exists a Mather measure $m_{0}$ whose support contains $(x, v)$ and a measure $m_{0}^{\prime}$ whose support contains $\left(x^{\prime}, v^{\prime}\right)$. But then $\left(m_{0}+m_{0}^{\prime}\right) / 2$ is clearly a Mather measure whose support contains $\left\{(x, v),\left(x^{\prime}, v^{\prime}\right)\right\}$ and is itself included in the graph of a $K$-Lipschitz vector field. Assuming that $x$ and $x^{\prime}$ lie in the image $\theta\left(B_{1}\right)$ of a common chart (see Appendix), so that $(x, v)=d \theta(X, V)$ and $\left(x^{\prime}, v^{\prime}\right)=d \theta\left(X^{\prime}, V^{\prime}\right)$, we obtain

$$
\left\|V-V^{\prime}\right\| \leq K\left\|x-x^{\prime}\right\| .
$$


It follows that the restriction to $\tilde{\mathcal{M}}$ of the canonical projection $T M \rightarrow M$ is a bi-Lipschitz homeomorphism, or equivalently that the set $\tilde{\mathcal{M}}$ is contained in the graph of a Lipschitz vector field.

\section{Appendix. Notations and standing conventions}

- $M$ is a compact manifold of dimension $d$, and $\pi: T M \rightarrow M$ is the canonical projection.

- We denote by $\tau: T M \times[0, T] \rightarrow[0, T]$ or $M \times[0, T] \rightarrow[0, T]$ the projection on the second factor.

- If $N$ is any separable, complete, locally compact metric space (for example $M, M \times$ $[0, T], T M$ or $T M \times[0, T]))$ the sets $\mathcal{B}_{1}(N) \subset \mathcal{B}_{+}(N) \subset \mathcal{B}(N)$ are respectively the set of Borel probability measures, non-negative Borel finite measures, and finite Borel signed measures. If $C_{c}(N)$ is the set of continuous compactly supported functions on $N$, endowed with the topology of uniform convergence, then the space $\mathcal{B}(N)$ is identified with the set of continuous linear forms on $C_{c}(N)$ by the Riesz theorem. We will always endow the space $\mathcal{B}(N)$ with the weak* topology that we will also call the weak topology. Note that the set $\mathcal{B}_{1}(N)$ is compact if $N$ is. Prokhorov's theorem states that a sequence of probability measures $P_{n} \in \mathcal{B}_{1}(N)$ has a subsequence converging in $\mathcal{B}_{1}(N)$ for the weak* topology if for all $\epsilon>0$ there exists a compact set $K_{\epsilon}$ such that $P_{n}\left(N-K_{\epsilon}\right) \leq \epsilon$ for all $n \in \mathbb{N}$. See e.g. [35, 16, 9].

- Given two manifolds $N$ and $N^{\prime}$, a Borel map $F: N \rightarrow N^{\prime}$, and a measure $\mu \in \mathcal{B}(N)$, we define the push-forward $F_{\sharp} \mu$ of $\mu$ by $F$ as the unique measure on $N^{\prime}$ which satisfies

$$
F_{\sharp} \mu(B)=\mu\left(F^{-1}(B)\right)
$$

for all Borel sets $B \in N$, or equivalently

$$
\int_{N^{\prime}} f d\left(F_{\sharp} \mu\right)=\int_{N} f \circ F d \mu
$$

for all continuous functions $f: N^{\prime} \rightarrow \mathbb{R}$.

- A family $\mu_{t}, t \in[0, T]$, of measures in $\mathcal{B}(N)$ is called measurable if the map $t \mapsto$ $\int_{N} f_{t} d \mu_{t}$ is Borel measurable for each $f \in C_{c}(N \times[0, T])$. We define the measure $\mu_{t} \otimes d t$ on $N \times[0, T]$ by

$$
\int_{N \times[0, T]} f d\left(\mu_{t} \otimes d t\right)=\int_{0}^{T} \int_{N} f_{t} d \mu_{t} d t
$$

for each $f \in C_{c}(N \times[0, T])$. The well-known desintegration theorem states that, if $\mu$ is a measure on $N \times[0, T]$ such that the projected measure on $[0, T]$ is the Lebesgue measure $d t$, then there exists a measurable family of measures $\mu_{t}$ on $N$ such that $\mu=$ $\mu_{t} \otimes d t$. 
- The set $\mathcal{K}\left(\mu_{0}, \mu_{T}\right)$ of transport plans is defined in Section 1.2

- The set $\mathcal{I}\left(\mu_{0}, \mu_{T}\right)$ of initial transport measures is defined in Section 2.1

- The set $\mathcal{M}\left(\mu_{0}, \mu_{T}\right)$ of transport measures is defined in Section 2.1

- The set $\mathcal{C}\left(\mu_{0}, \mu_{T}\right)$ of transport currents is defined in Section 2.2 .

- We fix, once and for all, a finite atlas $\Theta$ of $M$, formed by charts $\theta: B_{5} \rightarrow M$, where $B_{r}$ is the open ball of radius $r$ centered at zero in $\mathbb{R}^{d}$. We assume in addition that the sets $\theta\left(B_{1}\right), \theta \in \Theta$, cover $M$.

- We say that a vector field $X: M \rightarrow T M$ is $K$-Lipschitz if, for each chart $\theta \in \Theta$, the mapping $\Pi \circ(d \theta)^{-1} \circ X \circ \theta: B_{5} \rightarrow \mathbb{R}^{d}$ is $K$-Lipschitz on $B_{1}$, where $\Pi$ is the projection $B_{5} \times \mathbb{R}^{d} \rightarrow \mathbb{R}^{d}$.

- We mention the following results which are used throughout the paper. There exists a constant $C$ such that, if $A$ is a subset of $M$, and $X_{A}: A \rightarrow T M$ is a $K$-Lipschitz vector field, then there exists a $C K$-Lipschitz vector field $X$ on $M$ which extends $X_{A}$. In addition, if $A$ is a subset of $M \times[0, T]$ and $X_{A}: A \rightarrow T M$ is a $K$-Lipschitz vector field, then there exists a $C K$-Lipschitz vector field $X$ on $M \times[0, T]$ which extends $X_{A}$. If $A$ is a compact subset of $M \times[0, T]$ and $\left.X_{A}: A \cap M \times\right] 0, T[\rightarrow T M$ is a locally Lipschitz vector field (which is $K(\epsilon)$-Lipschitz on $A \cap M \times[\epsilon, T-\epsilon]$ ), then there exists a locally Lipschitz $(C K(\epsilon)$-Lipschitz on $M \times[\epsilon, T-\epsilon])$ vector field $X$ on $M \times] 0, T$ [ which extends $X_{A}$,

Acknowledgments. This paper results from the collaboration of the authors towards the end of the stay of the first author in EPFL for the academic year 2002-2003, supported by the Swiss National Science Foundation.

\section{References}

[1] Ambrosio, L.: Lecture notes on optimal transport problems. In: Mathematical Aspects of Evolving Interfaces (Funchal, 200), Lecture Notes in Math. 1812, Springer, 1-52 (2003). Zbl 1047.35001 MR 2011032

[2] Ambrosio, L.: Lecture notes on transport equation and Cauchy problem for BV vector fields and applications. (2004)

[3] Ambrosio, L., Gigli, N., Savaré, G.: Gradient Flows in Metric Spaces and in the Space of Probability Measures. Lectures in Math. ETH Zürich, Birkhäuser (2005) Zbl pre02152346 MR 2129498

[4] Ambrosio, L., Pratelli, A.: Existence and stability results in the $L^{1}$ theory of optimal transportation. In: Lecture Notes in Math. 1813, Springer, 123-160 (2003) Zbl 1065.49026 MR 2006307

[5] Bangert, V.: Minimal measures and minimizing closed normal one-currents. Geom. Funct. Anal. 9, 413-427 (1999) Zbl 0973.58004 MR 1708452

[6] Benamou, J.-D., Brenier, Y.: A computational fluid mechanics solution to the MongeKantorovich mass transfer problem. Numer. Math. 84, 375-393 (2000) Zbl 0968.76069 MR 1738163 
[7] Bernard, P.: The dynamics of pseudographs in convex Hamiltonian systems. Preprint

[8] Bernard, P., Buffoni, B.: The Monge problem for supercritical Mañé potential on compact manifolds. Preprint (2005)

[9] Billingsley, P.: Convergence of Probability Measures. 2nd ed., Wiley-Interscience (1999) Zbl 0944.60003 MR 1700749

[10] Brenier, Y.: Décomposition polaire et réarrangement monotone des champs de vecteurs. C. R. Acad. Sci. Paris Sér. I Math. 305, 805-808 (1987) Zbl 0652.26017 MR 0923203

[11] Brenier, Y.: Polar factorization and monotone rearrangement of vector-valued functions. Comm. Pure Appl. Math. 44, 375-417 (1991) Zbl 0738.46011 MR 1100809

[12] Brenier, Y.: Extended Monge-Kantorovich theory. In: Optimal Transportation and Applications (Martina Franca, 2001), Lecture Notes in Math. 1813, Springer, Berlin, 91-121 (2003) Zbl 1064.49036

[13] Cannarsa, P., Sinestrari, C.: Semiconcave Functions, Hamilton-Jacobi Equations and Optimal Control. Progr. Nonlinear Differential Equations Appl. 58, Birkhäuser (2004) Zbl pre02129788 MR 2041617

[14] De Pascale, L., Gelli, M. S., Granieri, L.: Minimal measures, one-dimensional currents and the Monge-Kantorovich problem. Calc. Var. Partial Differential Equations, to appear

[15] Carlier, G.: Duality and existence for a class of mass transportation problems and economic applications. Adv. Math. Economy 5, 1-21 (2003) Zbl pre02134650 MR 2160899

[16] Dudley, R. M.: Real Analysis and Probability. Cambridge Univ. Press (2002) Zbl 1023.60001 MR 1932358

[17] Evans, L. C., Gangbo, W.: Differential equation methods for the Monge-Kantorovich mass transfer problem. Mem. Amer. Math. Soc. 137, no. 653 (1999) Zbl 0920.49004 MR 1464149

[18] Evans, L. C., Gomes, D.: Linear programming interpretations of Mather's variational principle. ESAIM Control Optim. Calc. Var. 8, 693-702 (2002) Zbl pre01967389 MR 1932968

[19] Fathi, A.: Weak KAM Theorem in Lagrangian Dynamics. Preliminary version, Lyon (2001)

[20] Fathi, A., Siconolfi, A.: Existence of $C^{1}$ critical subsolutions of the Hamilton-Jacobi equation. Invent. Math. 155, 363-388 (2004) Zbl 1061.58008 MR 2031431

[21] Federer, H.: Geometric Measure Theory. Springer (1969) Zbl 0176.00801 MR 0257325

[22] Gangbo, W.: Habilitation thesis

[23] Gangbo, W., J. McCann, R.: The geometry of optimal transportation. Acta Math. 177, 113161 (1996) Zbl 0887.49017 MR 1440931

[24] Giaquinta, M., Modica, G., Souček, J.: Cartesian Currents in the Calculus of Variations I. Springer (1998) Zbl 0914.49001 MR 1645086

[25] Granieri, L.: On action minimizing measures for the Monge-Kantorovich problem. Preprint

[26] Kantorovich, L. V.: On the transfer of masses. Dokl. Akad. Nauk SSSR 37, 227-229 (1942) (in Russian); reprinted in: Zap. Nauchn. Semin. POMI 312, 11-144 (2004) Zbl 1080.49507 MR 2117876

[27] Kantorovich, L. V.: On a problem of Monge. Uspekhi Mat. Nauk 3, 225-226 (1948) (in Russian); reprinted in: Zap. Nauchn. Semin. POMI 312, 15-16 (2004) Zbl pre02213827 MR 2117877

[28] Loeper, G.: The reconstruction problem for the Euler-Poisson system in cosmology. Arch. Ration. Mech. Anal. 179, 153-216 (2006) Zbl pre05009583 MR 2209129

[29] Mañé, R.: Generic properties and problems of minimizing measures of Lagrangian systems. Nonlinearity 9, 273-310 (1996) Zbl 0886.58037 MR 1384478

[30] Mather, J. N.: Action minimizing invariant measures for positive definite Lagrangian systems. Math. Z. 207, 169-207 (1991) Zbl 0696.58027 MR 1109661 
[31] Monge, G.: Mémoire sur la théorie des déblais et des remblais. Hist. de l'Académie des Sciences de Paris, avec les Mémoires de Mathématiques et de Physique pour la même année, 666-704 (1781)

[32] Pratelli, A.: Equivalence between some definitions for the optimal mass transportation problem and for the transport density on manifolds. Ann. Mat. Pura Appl. 184, 215-238 (2005)

[33] Rachev, S. T., Rüschendorf, L.: Mass Transportation Problems. Vols. I and II, Springer (1998) Zbl 0990.60500 MR 1619170+ MR 1619171

[34] Ruelle, D., Sullivan, D.: Currents, flows and diffeomorphisms. Topology 14, 319-327 (1975) Zbl 0321.58019 MR 0415679

[35] Villani, C.: Topics in Optimal Transportation. Amer. Math. Soc., Providence, RI (2003) Zbl pre01909499 MR 1964483

[36] Wolansky, G.: Optimal transportation in the presence of a prescribed pressure field. (2004)

[37] Young, L. C.: Lectures on the Calculus of Variations and Optimal Control Theory. 2nd ed., \begin{tabular}{llll} 
Chelsea (1980) Zbl 0177.37801 & MR 0259704 \\
\hline
\end{tabular} 